\title{
MINIMIZING TOTAL VARIATION FLOW
}

\author{
F. ANDREU \\ Dept. de Análisis Matemático, Universitat de Valencia \\ 46100 Burjassot (Valencia), Spain \\ C. BAllester \\ Dept. de Tecnologia, Universitat Pompeu Fabra \\ La Rambla, 30-32, 08002 Barcelona, Spain \\ V. CAselles \\ Dept. de Tecnologia, Universitat Pompeu Fabra \\ La Rambla, 30-32, 08002 Barcelona, Spain

\section{J. M. MAzÓN} \\ Dept. de Análisis Matemático, Universitat de Valencia \\ 46100 Burjassot (Valencia), Spain \\ (Submitted by: Jerry Goldstein)
}

\begin{abstract}
We prove existence and uniqueness of weak solutions for the minimizing total variation flow with initial data in $L^{1}$. We prove that the length of the level sets of the solution, i.e., the boundaries of the level sets, decreases with time, as one would expect, and the solution converges to the spatial average of the initial datum as $t \rightarrow \infty$. We also prove that local maxima strictly decrease with time; in particular, flat zones immediately decrease their level. We display some numerical experiments illustrating these facts.
\end{abstract}

1. Introduction. Let $\Omega$ be a bounded set in $\mathbb{R}^{N}$ with Lipschitz-continuous boundary $\partial \Omega$. We are interested in the problem

$$
\begin{array}{ll}
\frac{\partial u}{\partial t}=\operatorname{div}\left(\frac{D u}{|D u|}\right) & \text { in } Q=(0, \infty) \times \Omega \\
\frac{\partial u}{\partial \eta}=0 & \text { on } S=(0, \infty) \times \partial \Omega
\end{array}
$$

Accepted for publication October 1999.

AMS Subject Classifications: 35K65, 35K55, 65M06. 


$$
u(0, x)=u_{0}(x) \quad \text { in } x \in \Omega
$$

where $u_{0} \in L^{1}(\Omega)$. This PDE appears when one uses the steepest descent method to minimize the total variation, a method introduced by L. Rudin and S. Osher [17], [18] in the context of image denoising and reconstruction. Then solving (1) amounts to regularizing or, in other words, to filtering the initial datum $u_{0}$. This filtering process has less destructive effect on the edges than filtering with a Gaussian, i.e., than solving the heat equation with initial condition $u_{0}$. In this context the given image $u_{0}$ is a function defined on a bounded, smooth or piecewise-smooth, open subset $\Omega$ of $\mathbb{R}^{N}$ typically, $\Omega$ will be a rectangle in $\mathbb{R}^{2}$. When dealing with the deconvolution or reconstruction problem one minimizes the total variation functional, i.e., the functional

$$
\text { Minimize } \int_{\Omega}|\nabla u|
$$

with some constraints, which models the process of image adquisition, including blur and noise. We shall not pursue this any longer, and we refer instead to [16], [17], [18], [11], [12], [10], [22], [23], [2], [20].

As argued in [1], the choice of Neumann boundary conditions is a natural choice in image processing. It corresponds to the reflection of the picture across the boundary and has the advantage of not imposing any value on the boundary and not creating edges on it.

We shall prove existence and uniqueness of solutions of (1) for initial data in $L^{1}(\Omega)$. To make precise our notion of solution let us recall several facts concerning functions of bounded variation.

A function $u \in L^{1}(\Omega)$ whose partial derivatives in the sense of distributions are measures with finite total variation in $\Omega$ is called a function of bounded variation. The class of such functions will be denoted by $B V(\Omega)$. Thus $u \in B V(\Omega)$ if and only if there are Radon measures $\mu_{1}, \ldots, \mu_{N}$ defined in $\Omega$ with finite total mass in $\Omega$ and

$$
\int_{\Omega} u D_{i} \varphi d x=-\int_{\Omega} \varphi d \mu_{i}
$$

for all $\varphi \in C_{0}^{\infty}(\Omega), i=1, \ldots, N$. Thus the gradient of $u$ is a vector-valued measure with finite total variation

$$
\|D u\|=\sup \left\{\int_{\Omega} u \operatorname{div} \varphi d x: \varphi \in C_{0}^{\infty}\left(\Omega, \mathbb{R}^{n}\right),|\varphi(x)| \leq 1 \text { for } x \in \Omega\right\} .
$$


The space $B V(\Omega)$ is endowed with the norm

$$
\|u\|_{B V}=\|u\|_{L^{1}(\Omega)}+\|D u\| .
$$

For further information concerning functions of bounded variation we refer to [15] and [24].

By $L_{w}^{1}(0, T, B V(\Omega))$ we denote the space of functions $w:[0, T] \rightarrow B V(\Omega)$ such that $w \in L^{1}((0, T) \times \Omega)$, the maps $t \in[0, T] \rightarrow\langle D w(t), \phi\rangle$ are measurable for every $\phi \in C_{0}^{1}\left(\Omega, \mathbb{R}^{N}\right)$ and such that $\int_{0}^{T}\|D w(t)\|<\infty$. Observe that, using (4), it follows easily that the map $t \in[0, T] \rightarrow\|D w(t)\|$ is measurable. Recall that $B V(\Omega)$ has a separable predual $G$, and the definition above amounts to say that $t \in[0, T] \rightarrow\langle D w(t), \phi\rangle$ is measurable for all $\phi \in G$ (see [3]). Now, from the density of $G$ in $B V(\Omega)^{*}$ in the topology $\sigma\left(B V(\Omega)^{*}, B V(\Omega)\right)$, and the fact that $\|D w(t)\| \in L^{1}(0, T)$, the measurability of the map $t \in[0, T] \rightarrow\langle D w(t), \phi\rangle$ follows for all $\phi \in B V(\Omega)^{*}$. Since we are not going to use this formulation below, we shall omit the details of its proof. To give the notion of solution we need the truncature operator $T_{k}(r)=\left[k-(k-|r|)^{+}\right] \operatorname{sign}(r), k \geq 0, r \in \mathbb{R}$.

Definition 1. A measurable function $u:(0, T) \times \Omega \rightarrow \mathbb{R}$ is a weak solution of $(1)$ in $(0, T) \times \Omega$ if $u \in C\left([0, T], L^{1}(\Omega)\right) \cap W_{l o c}^{1,1}\left(0, T ; L^{1}(\Omega)\right), T_{k}(u) \in$ $L_{w}^{1}(0, T ; B V(\Omega))$ for all $k>0$ and there exists $z \in L^{\infty}((0, T) \times \Omega)$ with $\|z\|_{\infty} \leq 1, u_{t}=\operatorname{div}(z)$ in $\mathcal{D}^{\prime}((0, T) \times \Omega)$ such that

$$
\int_{\Omega}\left(T_{k}(u(t))-w\right) u_{t}(t) \leq \int_{\Omega} z(t) \cdot \nabla w-\left\|D T_{k}(u(t))\right\|
$$

for every $w \in W^{1,1}(\Omega) \cap L^{\infty}(\Omega)$ and almost everywhere on $[0, T]$.

Our main result is the following:

Theorem 1. Let $u_{0} \in L^{1}(\Omega)$. Then there exists a unique weak solution of (1) in $(0, T) \times \Omega$ for every $T>0$ such that $u(0)=u_{0}$. Moreover, if $u(t), \hat{u}(t)$ are weak solutions corresponding to initial data $u_{0}, \hat{u}_{0}$, respectively, then

$$
\left\|(u(t)-\hat{u}(t))^{+}\right\|_{1} \leq\left\|\left(u_{0}-\hat{u}_{0}\right)^{+}\right\|_{1} \text { and }\|u(t)-\hat{u}(t)\|_{1} \leq\left\|u_{0}-\hat{u}_{0}\right\|_{1},
$$

for all $t \geq 0$.

To prove Theorem 1 we shall use the techniques of completely accretive operators [7] and Crandall-Liggett's semigroup generation theorem [13]. Let us recall the notion of completely accretive operators introduced in [7]. Let 
$\mathcal{M}(\Omega)$ be the space of measurable functions in $\Omega$. Given $u, v \in \mathcal{M}(\Omega)$, we shall write that

$$
u \ll v \text { if and only if } \int_{\Omega} j(u) d x \leq \int_{\Omega} j(v) d x
$$

for all $j \in J_{0}$ where

$$
J_{0}=\{j: \mathbb{R} \rightarrow[0, \infty] \text {, convex, l.s.c., } j(0)=0\}
$$

(l.s.c. is an abbreviation for lower semicontinuous function). Let $A$ be an operator (possibly multivalued) in $\mathcal{M}(\Omega)$, i.e., $A \subseteq \mathcal{M}(\Omega) \times \mathcal{M}(\Omega)$. We shall say that $A$ is completely accretive if

$$
u-\hat{u} \ll u-\hat{u}+\lambda(v-\hat{v}) \quad \text { for all } \lambda>0 \text { and all }(u, v),(\hat{u}, \hat{v}) \in A .
$$

Let $P_{0}=\left\{p \in C^{\infty}(\mathbb{R}): 0 \leq p^{\prime} \leq 1, \operatorname{supp}\left(p^{\prime}\right)\right.$ is compact and $\left.0 \notin \operatorname{supp}(p)\right\}$. If $A \subseteq L^{1}(\Omega) \times L^{1}(\Omega)$, then $A$ is completely accretive if and only if

$$
\int_{\Omega} p(u-\hat{u})(v-\hat{v}) \geq 0 \quad \text { for any } p \in P_{0},(u, v),(\hat{u}, \hat{v}) \in A .
$$

A completely accretive operator in $L^{1}(\Omega)$ is said to be $m$-completely accretive if $R(I+\lambda A)=L^{1}(\Omega)$ for any $\lambda>0$. In that case, by Crandall-Ligget's theorem, $A$ generates an order-preserving contraction semigroup in $L^{1}(\Omega)$ given by the exponential formula $e^{-t A} u_{0}=\lim _{n \rightarrow \infty}\left(I+\frac{t}{n} A\right)^{-n} u_{0}$ for any $u_{0} \in L^{1}(\Omega)$. Let us write $u(t)=e^{-t A} u_{0}$. Then $u \in C\left([0, T], L^{1}(\Omega)\right)$, for any $T>0$, and is a mild solution (a solution in the sense of semigroups [7]) of

$$
\frac{d u}{d t}+A u \ni 0,
$$

such that $u(0)=u_{0}$.

We shall use a stronger notion of solution of (12). We say that $v \in$ $C\left([0, T], L^{1}(\Omega)\right)$ is a strong solution of $(12)$ on $[0, T]$ if $v \in W_{l o c}^{1,1}\left((0, T), L^{1}(\Omega)\right)$ and $v^{\prime}(t)+A v(t) \ni 0$ for almost all $t \in(0, T)$. If $u_{0} \in D(A)=\left\{\bar{u} \in L^{1}(\Omega)\right.$ : $(\bar{u}, \bar{v}) \in A$, for some $\left.\bar{v} \in L^{1}(\Omega)\right\}$ (the domain of $A$ ) and $A$ is $m$-completely accretive, then $u \in W_{l o c}^{1,1}\left((0, T), L^{1}(\Omega)\right)$ and $u(t)$ is a strong solution of (12) on $(0, T)$, for all $T>0$.

In Section 2, we prove Theorem 1. For that, we shall associate a completely accretive operator $\mathcal{A}$ to the formal differential expression $-\operatorname{div}\left(\frac{D u}{|D u|}\right)$ 
together with Neumann boundary conditions. Then using Crandall-Liggett's semigroup generation theorem [13] we conclude that the abstract Cauchy problem in $L^{1}(\Omega)$

$$
\frac{d u}{d t}+\mathcal{A} u \ni 0, \quad u(0)=u_{0}
$$

has a unique strong solution $u \in C\left([0, T], L^{1}(\Omega)\right) \cap W_{l o c}^{1,1}\left(0, T ; L^{1}(\Omega)\right)(\forall T>$ 0 ) with initial datum $u(0)=u_{0}$. In Section 3 we shall prove that strong solutions of (13) coincide with weak solutions of (1). Section 4 is devoted to the asymptotic behavior of solutions of (1). In Section 5 we prove that, as one would expect, the length of the level curves (i.e., the boundaries of the level sets) of the solution $u(t, x)$ of (1) decreases with time. We also prove that the level of local maxima strictly decreases with time. Finally, in Section 6 we display some numerical experiments to illustrate the above properties.

2. The semigroup solution. Following [5], we define

$$
X(\Omega)=\left\{z \in L^{\infty}\left(\Omega, \mathbb{R}^{N}\right): \operatorname{div}(z) \in L^{1}(\Omega)\right\} .
$$

Let us introduce the following operator $\mathcal{A}$ in $L^{1}(\Omega):(u, v) \in \mathcal{A}$ if and only if $u, v \in L^{1}(\Omega), T_{k}(u) \in B V(\Omega)$ for all $k>0$ and there exists $z \in X(\Omega)$ with $\|z\|_{\infty} \leq 1, v=-\operatorname{div}(z)$ in $\mathcal{D}^{\prime}(\Omega)$ such that

$$
\int_{\Omega}\left(w-T_{k}(u)\right) v \leq \int_{\Omega} z \cdot \nabla w d x-\left\|D T_{k}(u)\right\|,
$$

$\forall w \in W^{1,1}(\Omega) \cap L^{\infty}(\Omega), \forall k>0$.

Theorem 2. The operator $\mathcal{A}$ is m-completely accretive in $L^{1}(\Omega)$ with dense domain. For any $u_{0} \in L^{1}(\Omega)$ the semigroup solution $u(t)=e^{-t \mathcal{A}_{u_{0}}}$ is a strong solution of

$$
\frac{d u}{d t}+\mathcal{A} u \ni 0, \quad u(0)=u_{0} .
$$

Let us introduce the following auxiliary operator $A$ in $L^{1}(\Omega):(u, v) \in A$ if and only if $u \in B V(\Omega) \cap L^{\infty}(\Omega), v \in L^{1}(\Omega)$ and there exists $z \in X(\Omega)$ with $\|z\|_{\infty} \leq 1, v=-\operatorname{div}(z)$ in $\mathcal{D}^{\prime}(\Omega)$ such that

$$
\int_{\Omega}(w-u) v \leq \int_{\Omega} z \cdot \nabla w d x-\|D u\|
$$


for every $w \in W^{1,1}(\Omega) \cap L^{\infty}(\Omega)$.

The following proposition relates both operators $A$ and $\mathcal{A}$.

Proposition 1. The operator $\mathcal{A}$ is the closure in $L^{1}(\Omega)$ of the operator $A$.

To prove this proposition we need first to establish the following results.

Lemma 1. $L^{\infty}(\Omega) \subseteq R(I+A)$.

Proof. For any $p>1$ we consider the operator $A_{p}$ in $\mathrm{七}^{1}(\Omega)$ defined by $(u, v) \in A_{p}$ if and only if $u \in W^{1, p}(\Omega) \cap L^{\infty}(\Omega), v \in L^{1}(\Omega)$ and

$$
\int_{\Omega}(w-u) v \leq \int_{\Omega}|\nabla u|^{p-2} \nabla u \cdot \nabla(w-u)
$$

for every $w \in W^{1, p}(\Omega) \cap L^{\infty}(\Omega)$.

By [4], we know that $A_{p}$ is completely accretive in $L^{1}(\Omega)$ and

$$
L^{\infty}(\Omega) \subseteq R\left(I+A_{p}\right) \quad(\text { for any } p>1) .
$$

Let $v \in L^{\infty}(\Omega)$. We find $u \in B V(\Omega) \cap L^{\infty}(\Omega)$ such that $(u, v-u) \in A$; i.e., there is $z \in X(\Omega)$ with $\|z\|_{\infty} \leq 1$ such that $v-u=-\operatorname{div}(z)$ and

$$
\int_{\Omega}(w-u)(v-u) \leq \int_{\Omega} z \cdot \nabla w d x-\|D u\|
$$

for every $w \in W^{1,1}(\Omega) \cap L^{\infty}(\Omega)$. Now, using (16), we know that for any $p>1$ there is $u_{p} \in W^{1, p}(\Omega) \cap L^{\infty}(\Omega)$ such that $\left(u_{p}, v-u_{p}\right) \in A_{p}$. Hence

$$
\int_{\Omega}\left(w-u_{p}\right)\left(v-u_{p}\right) \leq \int_{\Omega}\left|\nabla u_{p}\right|^{p-2} \nabla u_{p} \cdot \nabla\left(w-u_{p}\right),
$$

for every $w \in W^{1, p}(\Omega) \cap L^{\infty}(\Omega)$. Since $A_{p}$ is completely accretive, we have

$$
\left\|u_{p}\right\|_{\infty}=\left\|\left(I+A_{p}\right)^{-1} v\right\|_{\infty} \leq\|v\|_{\infty} .
$$

Now, given $\varphi \in W^{1, p}(\Omega) \cap L^{\infty}(\Omega)$ and taking $w=u_{p} \pm \varphi$ in (18) we get

$$
\int_{\Omega}\left|\nabla u_{p}\right|^{p-2} \nabla u_{p} \cdot \nabla \varphi=\int_{\Omega}\left(v-u_{p}\right) \varphi
$$

If we take $\varphi=u_{p}$ in (20) and taking (19) into account we obtain the estimate

$$
\int_{\Omega}\left|\nabla u_{p}\right|^{p}=\int_{\Omega}\left(v-u_{p}\right) u_{p} \leq M_{1}
$$


for any $p>1$, where $M_{1}$ depends on $\|v\|_{\infty}$ and the measure of $\Omega$. Using Holder's inequality we also have that

$$
\int_{\Omega}\left|\nabla u_{p}\right| \leq M_{2}, \quad \forall p>1
$$

where $M_{2}$ does not depend on $p$. Thus, $\left\{u_{p}\right\}_{p>1}$ is bounded in $W^{1,1}(\Omega)$ and we may extract a subsequence such that $u_{p}$ converges in $L^{1}(\Omega)$ and almost everywhere to some $u \in L^{1}(\Omega)$ as $p \rightarrow 1+$. Now, by (19) and (22), we have that $u \in B V(\Omega) \cap L^{\infty}(\Omega)$.

Let us prove that $\left\{\left|\nabla u_{p}\right|^{p-2} \nabla u_{p}\right\}_{p>1}$ is weakly relatively compact in $L^{1}\left(\Omega, \mathbb{R}^{N}\right)$. For that, using $(21)$ we observe that

$$
\int_{\Omega}\left|\nabla u_{p}\right|^{p-1} \leq\left(\int_{\Omega}\left|\nabla u_{p}\right|^{p}\right)^{\frac{p-1}{p}} \operatorname{meas}(\Omega)^{\frac{1}{p}} \leq M_{3},
$$

where $M_{3}$ does not depend on $p$. On the other hand, for any measurable subset $E \subseteq \Omega$,

$$
\left.\left.\left|\int_{E}\right| \nabla u_{p}\right|^{p-2} \nabla u_{p}\left|\leq \int_{E}\right| \nabla u_{p}\right|^{p-1} \leq M_{1}^{\frac{p-1}{p}} \operatorname{meas}(E)^{\frac{1}{p}} .
$$

Thus, $\left\{\left|\nabla u_{p}\right|^{p-2} \nabla u_{p}\right\}_{p>1}$, being bounded and equi-integrable in $L^{1}\left(\Omega, \mathbb{R}^{N}\right)$, is weakly relatively compact in $L^{1}\left(\Omega, \mathbb{R}^{N}\right)$. We may assume that

$$
\left|\nabla u_{p}\right|^{p-2} \nabla u_{p} \rightarrow z \quad \text { as } p \rightarrow 1, \text { weakly in } L^{1}\left(\Omega, \mathbb{R}^{N}\right) .
$$

Taking $\varphi \in C_{0}^{\infty}(\Omega)$ in $(20)$ and letting $p \rightarrow 1$, we obtain

$$
\int_{\Omega}(v-u) \varphi=\int_{\Omega} z \cdot \nabla \varphi
$$

that is, $v-u=-\operatorname{div}(z)$ in $\mathcal{D}^{\prime}(\Omega)$. Let us prove that $\|z\|_{\infty} \leq 1$. For any $k>0$, let $B_{p, k}=\left\{x \in \Omega:\left|\nabla u_{p}(x)\right|>k\right\}$. As a consequence of (21) we have that

$$
\operatorname{meas}\left(B_{p, k}\right) \leq \frac{M_{1}}{k^{p}} \quad \text { for every } p>1, k>0 .
$$

As above, there is some $g_{k} \in L^{1}\left(\Omega, \mathbb{R}^{N}\right)$ such that $\left|\nabla u_{p}\right|^{p-2} \nabla u_{p} \chi_{B_{p, k}} \rightarrow g_{k}$ weakly in $L^{1}\left(\Omega, \mathbb{R}^{N}\right)$ as $p \rightarrow 1$. Now for any $\phi \in L^{\infty}\left(\Omega, \mathbb{R}^{N}\right)$ with $\|\phi\|_{\infty} \leq 1$, we easily prove that

$$
\left.\left|\int_{\Omega}\right| \nabla u_{p}\right|^{p-2} \nabla u_{p} \cdot \phi \chi_{B_{p, k}} \mid \leq \frac{M_{1}}{k}
$$


Letting $p \rightarrow 1$, we get that

$$
\int_{\Omega}\left|g_{k}\right| \leq \frac{M_{1}}{k} \quad \text { for every } k>0 .
$$

Since we have that $\left.|| \nabla u_{p}\right|^{p-2} \nabla u_{p} \chi_{\Omega \backslash B_{p, k}} \mid \leq k^{p-1}$ for any $p>1$, letting $p \rightarrow 1$, we obtain that $\left|\nabla u_{p}\right|^{p-2} \nabla u_{p} \chi_{\Omega \backslash B_{p, k}}$ weakly converges in $L^{1}\left(\Omega, \mathbb{R}^{N}\right)$ to some function $f_{k} \in L^{1}\left(\Omega, \mathbb{R}^{N}\right)$ such that $\left\|f_{k}\right\|_{\infty} \leq 1$. Hence, for any $k>0$, we may write $z=f_{k}+g_{k}$ with $\left\|f_{k}\right\|_{\infty} \leq 1$ and $g_{k}$ satisfying (25). It follows that $\|z\|_{\infty} \leq 1$.

Now, to prove (17), let $w \in W^{1,1}(\Omega) \cap L^{\infty}(\Omega)$ and let $w_{n} \in C^{\infty}(\bar{\Omega})$ be such that $w_{n} \rightarrow w$ in $W^{1,1}(\Omega)$ as $n \rightarrow \infty$. Using $w_{n}$ as a test function in (18) we may write

$$
\int_{\Omega}\left(w_{n}-u_{p}\right)\left(v-u_{p}\right) \leq \int_{\Omega}\left|\nabla u_{p}\right|^{p-2} \nabla u_{p} \cdot \nabla\left(w_{n}-u_{p}\right) .
$$

Hence,

$$
\int_{\Omega}\left(w_{n}-u_{p}\right)\left(v-u_{p}\right)+\int_{\Omega}\left|\nabla u_{p}\right|^{p} \leq \int_{\Omega}\left|\nabla u_{p}\right|^{p-2} \nabla u_{p} \cdot \nabla w_{n} .
$$

Now, letting $p \rightarrow 1$ in (26) we obtain

$$
\int_{\Omega}\left(w_{n}-u\right)(v-u)+\|D u\| \leq \int_{\Omega} z \cdot \nabla w_{n}
$$

Let us finally let $n \rightarrow \infty$ to get (17).

Lemma 2. $\overline{D(A)}^{L^{1}(\Omega)}=L^{1}(\Omega)$.

Proof. We prove that $C_{0}^{\infty}(\Omega) \subseteq \overline{D(A)}^{L^{1}(\Omega)}$. Let $v \in C_{0}^{\infty}(\Omega)$. By Lemma 1 , $v \in R\left(I+\frac{1}{n} A\right)$ for all $n \in \mathbb{N}$. Thus, for each $n \in \mathbb{N}$ there exists $u_{n} \in D(A)$ such that $\left(u_{n}, n\left(v-u_{n}\right)\right) \in A$ and, in consequence, there exists some $z_{n} \in$ $X(\Omega)$ with $\left\|z_{n}\right\|_{\infty} \leq 1, n\left(v-u_{n}\right)=-\operatorname{div}\left(z_{n}\right)$ in $\mathcal{D}^{\prime}(\Omega)$ such that

$$
\int_{\Omega}\left(w-u_{n}\right) n\left(v-u_{n}\right) \leq \int_{\Omega} z_{n} \cdot \nabla w-\left\|D u_{n}\right\| \leq \int_{\Omega}|\nabla w|
$$

for every $w \in W^{1,1}(\Omega) \cap L^{\infty}(\Omega)$. Taking $w=v$ we have that

$$
\int_{\Omega}\left(v-u_{n}\right)^{2} \leq \frac{1}{n} \int_{\Omega}|\nabla v| .
$$

Letting $n \rightarrow \infty$, it follows that $u_{n} \rightarrow v$ in $L^{2}(\Omega)$. Therefore $v \in \overline{D(A)} L^{1}(\Omega)$. 
Lemma 3. If $u \in B V(\Omega)$ then $T_{k}(u), G_{k}(u) \in B V(\Omega)$ where $G_{k}(r)=r-$ $T_{k}(r), k \geq 0, r \in \mathbb{R}$. Moreover, $\|D u\|=\left\|D T_{k}(u)\right\|+\left\|D G_{k}(u)\right\|$ for any $k \geq 0$.

Proof. Let $u_{n} \in W^{1,1}(\Omega)$ be such that $u_{n} \rightarrow u$ in $L^{1}(\Omega)$ and $\int_{\Omega}\left|\nabla u_{n}\right| \rightarrow$ $\|D u\|$ as $n \rightarrow \infty$. Since $T_{k}\left(u_{n}\right) \rightarrow T_{k}(u)$ in $L^{1}(\Omega)$, then

$$
\left\|D T_{k}(u)\right\| \leq \liminf _{n \rightarrow \infty}\left\|\nabla T_{k}\left(u_{n}\right)\right\| \leq \liminf _{n \rightarrow \infty}\left\|\nabla u_{n}\right\| \leq\|D u\| .
$$

Hence, $T_{k}(u), G_{k}(u) \in B V(\Omega)$. Since $u=T_{k}(u)+G_{k}(u)$ we have that $\|D u\| \leq\left\|D T_{k}(u)\right\|+\left\|D G_{k}(u)\right\|$. On the other hand, since $u_{n} \in W^{1,1}(\Omega)$ we have that

$$
\int_{\Omega}\left|\nabla u_{n}\right|=\int_{\Omega}\left|\nabla T_{k}\left(u_{n}\right)\right|+\int_{\Omega}\left|\nabla G_{k}\left(u_{n}\right)\right|
$$

for any $k \geq 0$. Let $u_{n_{i}}$ be a subsequence of $u_{n}$ such that

$$
\liminf _{n \rightarrow \infty} \int_{\Omega}\left|\nabla T_{k}\left(u_{n}\right)\right|=\lim _{i \rightarrow \infty} \int_{\Omega}\left|\nabla T_{k}\left(u_{n_{i}}\right)\right| .
$$

Then we also have that

$$
\lim _{i \rightarrow \infty}\left(\int_{\Omega}\left|\nabla u_{n_{i}}\right|-\int_{\Omega}\left|\nabla T_{k}\left(u_{n_{i}}\right)\right|\right)=\lim _{i \rightarrow \infty} \int_{\Omega}\left|\nabla G_{k}\left(u_{n_{i}}\right)\right| .
$$

Hence

$$
\begin{aligned}
\|D u\| & =\lim _{i \rightarrow \infty} \int_{\Omega}\left|\nabla u_{n_{i}}\right|=\lim _{i \rightarrow \infty} \int_{\Omega}\left|\nabla T_{k}\left(u_{n_{i}}\right)\right|+\lim _{i \rightarrow \infty} \int_{\Omega}\left|\nabla G_{k}\left(u_{n_{i}}\right)\right| \\
& \geq\left\|D T_{k}(u)\right\|+\left\|D G_{k}(u)\right\| .
\end{aligned}
$$

Proof of Proposition 1. If $(u, v) \in \mathcal{A}$, then $\left(T_{k}(u), v\right) \in A$, for any $k>0$. Hence $(u, v) \in \bar{A}$. Conversely, let $(u, v) \in \bar{A}$ and let $\left(u_{n}, v_{n}\right) \in A$ be such that $u_{n} \rightarrow u, v_{n} \rightarrow v$ in $L^{1}(\Omega)$ as $n \rightarrow \infty$. Since $\left(u_{n}, v_{n}\right) \in A, u_{n} \in$ $B V(\Omega) \cap L^{\infty}(\Omega), v_{n} \in L^{1}(\Omega)$ and there exists $z_{n} \in X(\Omega),\left\|z_{n}\right\|_{\infty} \leq 1$ with $v_{n}=-\operatorname{div}\left(z_{n}\right)$ in $\mathcal{D}^{\prime}(\Omega)$ such that

$$
\int_{\Omega}\left(w-u_{n}\right) v_{n} \leq \int_{\Omega} z_{n} \cdot \nabla w-\left\|D u_{n}\right\|
$$

for every $w \in W^{1,1}(\Omega) \cap L^{\infty}(\Omega)$. Let $k>0$. Now, since $G_{k}\left(u_{n}\right) \in B V(\Omega)$, there exists a sequence $u_{m, n} \in W^{1,1}(\Omega) \cap L^{\infty}(\Omega)$ such that $\left\|u_{m, n}\right\|_{\infty} \leq M_{n}$ 
for all $m, n \in \mathbb{N}, u_{m, n} \rightarrow G_{k}\left(u_{n}\right)$ in $L^{1}(\Omega)$ and $\int_{\Omega}\left|\nabla u_{m, n}\right| \rightarrow|| D G_{k}\left(u_{n}\right) \|$ as $m \rightarrow \infty$. Let $w \in W^{1,1}(\Omega) \cap L^{\infty}(\Omega)$. Using $w+u_{m, n}$ as a test function in (27) we obtain

$$
\begin{aligned}
\int_{\Omega}\left(w+u_{m, n}-u_{n}\right) v_{n} & \leq \int_{\Omega} z_{n} \cdot\left(\nabla w+\nabla u_{m, n}\right)-\left\|D u_{n}\right\| \\
& \leq \int_{\Omega} z_{n} \cdot \nabla w+\int_{\Omega}\left|\nabla u_{m, n}\right|-\left\|D u_{n}\right\| .
\end{aligned}
$$

Letting $m \rightarrow \infty$ and using Lemma 3 we obtain

$$
\int_{\Omega}\left(w-T_{k}\left(u_{n}\right)\right) v_{n} \leq \int_{\Omega} z_{n} \cdot \nabla w-\left\|D T_{k}\left(u_{n}\right)\right\| .
$$

Taking $w=0$ in $(28)$ we have that

$$
\left\|D T_{k}\left(u_{n}\right)\right\| \leq \int_{\Omega} T_{k}\left(u_{n}\right) v_{n} \leq k \sup _{n}\left\|v_{n}\right\|_{1} .
$$

It follows that $T_{k}(u) \in B V(\Omega)$. Since $\left\|z_{n}\right\|_{\infty} \leq 1$ we may assume that $z_{n} \rightarrow z$ in the weak ${ }^{*}$ topology of $L^{\infty}\left(\Omega, \mathbb{R}^{N}\right)$ with $\|z\|_{\infty} \leq 1$. Now, letting $n \rightarrow \infty$ in (28) we obtain that

$$
\int_{\Omega}\left(w-T_{k}(u)\right) v \leq \int_{\Omega} z \cdot \nabla w-\left\|D T_{k}(u)\right\| .
$$

To prove Theorem 2 we need to use test functions in $B V(\Omega) \cap L^{\infty}(\Omega)$. The next lemma shows that this is indeed possible. We shall need several results from [5]. If $z \in X(\Omega)$ and $w \in B V(\Omega) \cap L^{\infty}(\Omega)$ we define the functional $(z, D w): C_{0}^{\infty}(\Omega) \rightarrow \mathbb{R}$ by the formula

$$
\langle(z, D w), \varphi\rangle=-\int_{\Omega} w \varphi \operatorname{div}(z) d x-\int_{\Omega} w z \cdot \nabla \varphi d x .
$$

Then $(z, D w)$ is a Radon measure in $\Omega$,

$$
\int_{\Omega}(z, D w)=\int_{\Omega} z \cdot \nabla w d x
$$

for all $w \in W^{1,1}(\Omega) \cap L^{\infty}(\Omega)$ and

$$
\left|\int_{B}(z, D w)\right| \leq \int_{B}|(z, D w)| \leq\|z\|_{\infty} \int_{B}\|D w\|
$$


for any Borel set $B \subseteq \Omega$. Moreover, $(z, D w)$ is absolutely continuous with respect to $\|D w\|$ with Radon-Nikodym derivative $\theta(z, D w, x)$, which is a $\|D w\|$ measurable function from $\Omega$ to $\mathbb{R}$ such that

$$
\int_{B}(z, D w)=\int_{B} \theta(z, D w, x)\|D w\|
$$

for any Borel set $B \subseteq \Omega$. We also have that

$$
\|\theta(z, D w, \cdot)\|_{L^{\infty}(\Omega,\|D w\|)} \leq\|z\|_{L^{\infty}\left(\Omega, \mathbb{R}^{N}\right)} .
$$

Lemma 4. We have the following characterization of the operator $\mathcal{A}$ :

$(u, v) \in \mathcal{A} \quad$ if and only if $u, v \in L^{1}(\Omega), T_{k}(u) \in B V(\Omega)$ for all $k>0$ and there exists $z \in X(\Omega)$ with $\|z\|_{\infty} \leq 1, v=-\operatorname{div}(z)$ in $\mathcal{D}^{\prime}(\Omega)$ such that

$$
\int_{\Omega}\left(w-T_{k}(u)\right) v \leq \int_{\Omega}(z, D w)-\left\|D T_{k}(u)\right\|,
$$

$\forall w \in B V(\Omega) \cap L^{\infty}(\Omega), \forall k>0$. Moreover, we have that

i) $\int_{\Omega}\left(z, D T_{k}(u)\right)=\left\|D T_{k}(u)\right\|$, for all $k>0$,

ii) $\int_{\Omega} v T_{k}(u)=\left\|D T_{k}(u)\right\|$, for all $k>0$,

iii) $\int_{\Omega} w v=\int_{\Omega}(z, D w)$, for all $w \in B V(\Omega) \cap L^{\infty}(\Omega)$.

Remark. As a consequence we also have the following characterization of $\mathcal{A}:(u, v) \in \mathcal{A}$ if and only if $u, v \in L^{1}(\Omega), T_{k}(u) \in B V(\Omega)$ for all $k>0$ and there exists $z \in X(\Omega)$ with $\|z\|_{\infty} \leq 1, v=-\operatorname{div}(z)$ in $\mathcal{D}^{\prime}(\Omega)$ such that $\int_{\Omega}\left(w-T_{k}(u)\right) v=\int_{\Omega}\left(z, D w-D T_{k}(u)\right), \forall w \in B V(\Omega) \cap L^{\infty}(\Omega), \forall k>0$.

Proof. Let $\mathcal{B}$ be the operator defined in the statement of the lemma. Since $\int_{\Omega}(z, D w)=\int_{\Omega} z \cdot \nabla w$ when $w \in W^{1,1}(\Omega) \cap L^{\infty}(\Omega)$ we have that $\mathcal{B} \subseteq \mathcal{A}$. Now, let $(u, v) \in \mathcal{A}$. Let $z \in X(\Omega)$ with $\|z\|_{\infty} \leq 1, v=-\operatorname{div}(z)$ in $\mathcal{D}^{\prime}(\Omega)$ such that

$$
\int_{\Omega}\left(\varphi-T_{k}(u)\right) v \leq \int_{\Omega} z \cdot \nabla \varphi-\left\|D T_{k}(u)\right\|
$$

$\forall \varphi \in W^{1,1}(\Omega) \cap L^{\infty}(\Omega), \forall k>0$. Let $w \in B V(\Omega) \cap L^{\infty}(\Omega)$. Using Lemmas 5.2 and 1.8 in [5] we know that there exists a sequence $w_{n} \in C^{\infty}(\Omega) \cap B V(\Omega)$ such that

$$
\begin{aligned}
& w_{n} \rightarrow w \quad \text { in } L^{1}(\Omega), \quad \int_{\Omega}\left|\nabla w_{n}\right| \rightarrow\|D w\|, \\
& \int_{\Omega} z \cdot \nabla w_{n}=\int_{\Omega}\left(z, D w_{n}\right) \rightarrow \int_{\Omega}(z, D w) .
\end{aligned}
$$


Using $\varphi=w_{n}$ as test function in (35) we may write

$$
\int_{\Omega}\left(w_{n}-T_{k}(u)\right) v \leq \int_{\Omega} z \cdot \nabla w_{n}-\left\|D T_{k}(u)\right\| .
$$

Letting $n \rightarrow \infty$ and taking (36) we obtain

$$
\int_{\Omega}\left(w-T_{k}(u)\right) v \leq \int_{\Omega}(z, D w)-\left\|D T_{k}(u)\right\| .
$$

We conclude that $(u, v) \in \mathcal{B}$ and, therefore, $\mathcal{A}=\mathcal{B}$.

Now, taking $w=T_{k}(u)$ in (34) we obtain $0 \leq \int_{\Omega}\left(z, D T_{k}(u)\right)-\left\|D T_{k}(u)\right\|$. Thus,

$$
\int_{\Omega}\left(z, D T_{k}(u)\right) \leq\|z\|_{\infty}\left\|D T_{k}(u)\right\| \leq\left\|D T_{k}(u)\right\| \leq \int_{\Omega}\left(z, D T_{k}(u)\right),
$$

and we obtain $i$ ). To prove $i i$ ) we take $w=0$ in (34) to obtain

$$
\left\|D T_{k}(u)\right\| \leq \int_{\Omega} v T_{k}(u)
$$

and then $w=2 T_{k}(u)$ to obtain, using $\left.i\right)$,

$$
\int_{\Omega} v T_{k}(u) \leq 2 \int_{\Omega}\left(z, D T_{k}(u)\right)-\left\|D T_{k}(u)\right\|=\left\|D T_{k}(u)\right\| .
$$

After using $i i)$ in (34) we may write $\int_{\Omega} w v \leq \int_{\Omega}(z, D w)$ for any $w \in B V(\Omega) \cap$ $L^{\infty}(\Omega)$. Since the same inequality holds for $-w \in B V(\Omega) \cap L^{\infty}(\Omega)$ we obtain iii).

Remark. As a consequence of $i)$ we have that $\theta\left(z, D T_{k}(u), x\right)=1$ almost everywhere with respect to the measure $\left\|D T_{k}(u)\right\|$. In case that $z \in C\left(\Omega, \mathbb{R}^{N}\right)$, this implies that $z(x) \cdot \frac{D T_{k}(u)}{\left\|D T_{k}(u)\right\|}=1,\left\|D T_{k}(u)\right\|$-almost everywhere where $\frac{D T_{k}(u)}{\left\|D T_{k}(u)\right\|}$ denotes the density of $D T_{k}(u)$ with respect to $\left\|D T_{k}(u)\right\|$. Heuristically, this amounts to saying that $z=\frac{D u}{\|D u\|}$. When $z$ is not continuous we have that $z(x) \cdot \frac{D T_{k}(u)}{\left\|D T_{k}(u)\right\|}=1,\left\|\nabla T_{k}(u)\right\|$-almost everywhere where $\left\|\nabla T_{k}(u)\right\|$ denotes the absolutely continuous part of $\left\|D T_{k}(u)\right\|$ with respect to the Lebesgue measure in $\mathbb{R}^{N}([5])$. In particular, if $u \in W^{1,1}(\Omega) \cap L^{\infty}(\Omega)$ we have that $z(x) \cdot \frac{\nabla u}{\|\nabla u\|}=1,\|\nabla u\|$-almost everywhere. 
Proof of Theorem 2. Let $(u, v),(\hat{u}, \hat{v}) \in \mathcal{A}, p \in P_{0}$. We have to prove that

$$
\int_{\Omega} p(u-\hat{u})(v-\hat{v}) \geq 0 .
$$

Let $z, \hat{z} \in X(\Omega),\|z\|_{\infty} \leq 1,\|\hat{z}\|_{\infty} \leq 1$, be such that $v=-\operatorname{div}(z), \hat{v}=$ $-\operatorname{div}(\hat{z})$ and

$$
\begin{aligned}
& \int_{\Omega}\left(w-T_{k}(u)\right) v=\int_{\Omega}(z, D w)-\left\|D T_{k}(u)\right\|, \\
& \int_{\Omega}\left(w-T_{k}(\hat{u})\right) \hat{v}=\int_{\Omega}(\hat{z}, D w)-\left\|D T_{k}(\hat{u})\right\|,
\end{aligned}
$$

for any $w \in B V(\Omega) \cap L^{\infty}(\Omega)$ and any $k>0$. As observed in the previous remark, $\theta\left(z, D T_{k}(u), x\right)=1\left\|D T_{k}(u)\right\|$-almost everywhere, and, using Corollary 1.6 in [5], we obtain that

$$
\begin{aligned}
& \int_{B}\left(z, D T_{k}(u)\right)=\int_{B} \theta\left(z, D T_{k}(u), x\right)\left\|D T_{k}(u)\right\|=\int_{B}\left\|D T_{k}(u)\right\|, \\
& \left|\int_{B}\left(\hat{z}, D T_{k}(u)\right)\right| \leq \int_{B}\left\|D T_{k}(u)\right\|
\end{aligned}
$$

for any Borel set $B \subseteq \Omega$. Similarly,

$$
\int_{B}\left(\hat{z}, D T_{k}(\hat{u})\right)=\int_{B}\left\|D T_{k}(\hat{u})\right\|, \quad\left|\int_{B}\left(z, D T_{k}(\hat{u})\right)\right| \leq \int_{B}\left\|D T_{k}(\hat{u})\right\|
$$

for any Borel set $B \subseteq \Omega$. It follows that

$$
\int_{B}\left(z-\hat{z}, D\left(T_{k}(u)-T_{k}(\hat{u})\right)\right) \geq 0
$$

for any Borel set $B \subseteq \Omega$. This implies that

$\theta\left(z-\hat{z}, D\left(T_{k}(u)-T_{k}(\hat{u})\right), x\right) \geq 0 \quad\left\|D\left(T_{k}(u)-T_{k}(\hat{u})\right)\right\|$-almost everywhere.

Since, according to Proposition 2.8 in [5], we have that

$$
\theta\left(z-\hat{z}, D p\left(T_{k}(u)-T_{k}(\hat{u})\right), x\right)=\theta\left(z-\hat{z}, D\left(T_{k}(u)-T_{k}(\hat{u})\right), x\right)
$$

almost everywhere with respect to the measures $\left\|D\left(T_{k}(u)-T_{k}(\hat{u})\right)\right\|$ and $\left\|D p\left(T_{k}(u)-T_{k}(\hat{u})\right)\right\|$. We conclude that

$$
\theta\left(z-\hat{z}, D p\left(T_{k}(u)-T_{k}(\hat{u})\right), x\right) \geq 0, \quad\left\|D p\left(T_{k}(u)-T_{k}(\hat{u})\right)\right\| \text { a.e. }
$$


Taking $w=T_{k}(u)+p\left(T_{k}(u)-T_{k}(\hat{u})\right)$ in $(39)$ and $w=T_{k}(\hat{u})-p\left(T_{k}(u)-T_{k}(\hat{u})\right)$ in (40), adding both terms, and using (41), we obtain

$$
\begin{aligned}
& \int_{\Omega} p\left(T_{k}(u)-T_{k}(\hat{u})\right)(v-\hat{v})=\int_{\Omega}\left(z-\hat{z}, D p\left(T_{k}(u)-T_{k}(\hat{u})\right)\right) \\
& =\int_{\Omega} \theta\left(z-\hat{z}, D p\left(T_{k}(u)-T_{k}(\hat{u})\right), x\right)\left\|D p\left(T_{k}(u)-T_{k}(\hat{u})\right)\right\| \geq 0 .
\end{aligned}
$$

The inequality (38) follows by letting $k \rightarrow \infty$. Therefore $\mathcal{A}$ is completely accretive. Since $\mathcal{A}$ is an extension of $A$, using Proposition 1 we obtain that $R(I+\mathcal{A})$ is dense in $L^{1}(\Omega)$. It follows that $\mathcal{A}$ is $m$-completely accretive in $L^{1}(\Omega)([7])$. By Crandall-Ligget's theorem, $\mathcal{A}$ generates a contraction semigroup in $L^{1}(\Omega)$ given by the exponential formula

$$
e^{-t \mathcal{A}} u_{0}=\lim _{n \rightarrow \infty}\left(I+\frac{t}{n} \mathcal{A}\right)^{-n} u_{0} \quad \text { for any } u_{0} \in L^{1}(\Omega)
$$

The function $u(t)=e^{-t \mathcal{A}_{u_{0}}}$ is a mild solution (a solution in the sense of semigroups [7]) of

$$
\frac{d u}{d t}+\mathcal{A} u \ni 0, \quad u(0)=u_{0} .
$$

To prove that $u(t)$ is a strong solution of (42) we shall use the regularizing effect due to the homogeneity of the operator $\mathcal{A}[6]$. Let us first observe that

$$
\text { if }(u, v) \in \mathcal{A} \text { and } \lambda>0 \text {, then }(\lambda u, v) \in \mathcal{A} \text {. }
$$

Indeed, let $(u, v) \in \mathcal{A}$ and let $z \in X(\Omega)$ with $\|z\|_{\infty} \leq 1, v=-\operatorname{div}(z)$ in $\mathcal{D}^{\prime}(\Omega)$ satisfying

$$
\int_{\Omega}\left(w-T_{k}(u)\right) v \leq \int_{\Omega}(z, D w)-\left\|D T_{k}(u)\right\|,
$$

$\forall w \in B V(\Omega) \cap L^{\infty}(\Omega), \forall k>0$. Then, take as test function in (44) $w+$ $T_{k}(u)-T_{k}(\lambda u)$ instead of $w \in B V(\Omega) \cap L^{\infty}(\Omega)$ to obtain

$$
\int_{\Omega}\left(w-T_{k}(\lambda u)\right) v \leq \int_{\Omega}(z, D w)-\left\|D T_{k}(\lambda u)\right\| .
$$

In other words, $(\lambda u, v) \in \mathcal{A}$. From (43) it follows immediately that

$$
\frac{1}{\lambda}(I+\lambda \mu \mathcal{A})^{-1} u_{0}=(I+\mu \mathcal{A})^{-1}\left(\frac{1}{\lambda} u_{0}\right)
$$


for any $\lambda, \mu>0$ and any $u_{0} \in L^{1}(\Omega)$. Iterating (45) and taking $\mu=\frac{t}{n}$ we obtain

$$
\left(I+\frac{t}{n} \mathcal{A}\right)^{-n}\left(\frac{1}{\lambda} u_{0}\right)=\frac{1}{\lambda}\left(I+\lambda \frac{t}{n} \mathcal{A}\right)^{-n} u_{0}
$$

for any $\lambda>0, n \in \mathbb{N}$ and $u_{0} \in L^{1}(\Omega)$. Writing $S(t)=e^{-t \mathcal{A}}$ and letting $n \rightarrow \infty$ in (46) we may write

$$
S(t)\left(\frac{1}{\lambda} u_{0}\right)=\frac{1}{\lambda} S(\lambda t) u_{0},
$$

for any $\lambda>0$ and any $u_{0} \in L^{1}(\Omega)$. Now, let $u_{0} \in L^{1}(\Omega)$ and $u(t)=S(t) u_{0}$. Since $\mathcal{A}$ is $m$-completely accretive in $L^{1}(\Omega), u(t)$ will be a strong solution of (42) once we know that $S(t) u_{0} \in D(\mathcal{A})$ for all $t>0$ ([7], Theorem 4.2). From the proof of Theorem 4.2 in [7] it is sufficient to prove that, given $t>0$, for some sequence $t_{n} \downarrow 0$

$$
\left\{\frac{S\left(t+t_{n}\right) u_{0}-S(t) u_{0}}{t_{n}}\right\}_{n=1}^{\infty} \text { is weakly convergent in } L^{1}(\Omega) .
$$

Fix $t>0$ and let $h>0, \lambda=1+\frac{h}{t}$. Using (47) we have that

$$
\begin{aligned}
S(t+h) u_{0}-S(t) u_{0} & =S(\lambda t) u_{0}-S(t) u_{0}=\lambda S(t)\left(\frac{1}{\lambda} u_{0}\right)-S(t) u_{0} \\
& =\lambda\left[S(t)\left(\frac{1}{\lambda} u_{0}\right)-S(t) u_{0}\right]+(\lambda-1) S(t) u_{0} .
\end{aligned}
$$

From this, it follows that

$$
\left|S(t+h) u_{0}-S(t) u_{0}\right| \leq \lambda\left|S(t)\left(\frac{1}{\lambda} u_{0}\right)-S(t) u_{0}\right|+|\lambda-1|\left|S(t) u_{0}\right| .
$$

The complete accretivity of $\mathcal{A}$ implies that $S(t)\left(\frac{1}{\lambda} u_{0}\right)-S(t) u_{0}<<\frac{1}{\lambda} u_{0}-u_{0}$, $S(t) u_{0}<<u_{0}$. Since $u<<v, u, v \in \mathcal{M}(\Omega)$ implies that $\alpha u<<\alpha v, \alpha>0$, and $|u|<<|v|$, the previous relations in turn imply that

$$
\begin{aligned}
& \lambda\left|S(t)\left(\frac{1}{\lambda} u_{0}\right)-S(t) u_{0}\right|<<(\lambda-1)\left|u_{0}\right|, \\
& (\lambda-1)\left|S(t) u_{0}\right|<<(\lambda-1)\left|u_{0}\right| .
\end{aligned}
$$

Since the set $\left\{f \in \mathcal{M}(\Omega): f<<(\lambda-1)\left|u_{0}\right|\right\}$ is convex we deduce from (49) and (50) that $\left|S(t+h) u_{0}-S(t) u_{0}\right|<<2(\lambda-1)\left|u_{0}\right|=2 \frac{h}{t}\left|u_{0}\right|$; hence,

$$
\frac{\left|S(t+h) u_{0}-S(t) u_{0}\right|}{h}<<\frac{2}{t}\left|u_{0}\right| \text {. }
$$


Now, using Proposition 2.11 in [7] we conclude that $\left\{\frac{\left|S(t+h) u_{0}-S(t) u_{0}\right|}{h}\right\}_{h>0}$ is weakly compact in $L^{1}(\Omega)$ and (48) holds. Notice also that as a consequence of (51) we obtain

$$
\left|u^{\prime}(t)\right| \leq \frac{2}{t}\left|u_{0}\right|
$$

Remark. Let us mention another proof of the complete accretivity of $\mathcal{A}$. For that we consider the functional $\Phi: L^{1}(\Omega) \rightarrow(-\infty,+\infty]$ defined by

$$
\Phi(u)= \begin{cases}\int_{\Omega}|\nabla u| & \text { if } u \in W^{1,1}(\Omega) \\ +\infty & \text { if } u \notin W^{1,1}(\Omega) .\end{cases}
$$

Then, using Lemma 7.1 in ([7]) we know that the operator $\partial_{L^{1}(\Omega)} \Phi$ in $L^{1}(\Omega)$ defined by $(u, v) \in \partial_{L^{1}(\Omega)} \Phi$ if and only if $u \in W^{1,1}(\Omega), v \in L^{1}(\Omega)$, and $\Phi(w) \geq \Phi(u)-\int_{\Omega}(w-u) v, \forall w \in L^{1}(\Omega)$ such that $(w-u) v \in L^{1}(\Omega)$ is completely accretive in $L^{1}(\Omega)$. Now, the lower-semicontinuous envelope of the functional $\Phi$ is the functional $\Psi$ given by

$$
\Psi(u)= \begin{cases}\int_{\Omega}|\nabla u| & \text { if } u \in B V(\Omega) \\ +\infty & \text { if } u \notin B V(\Omega),\end{cases}
$$

and, using Lemma 7.5 in [7], we know that ${\overline{\partial_{L^{1}(\Omega)} \Psi}}^{L^{1}(\Omega)}$ is $m$-completely accretive in $L^{1}(\Omega)$. We obtain using Proposition 1 above and Proposition 3.4 in [7] that $\mathcal{A}={\overline{\partial_{L^{1}(\Omega)}}}^{L^{1}(\Omega)}$.

\section{Existence and uniqueness of weak solutions.}

Lemma 5. Let $u \in C\left([0, T], L^{1}(\Omega)\right) \cap W_{l o c}^{1,1}\left(0, T ; L^{1}(\Omega)\right)$ be the strong solution of (15) with initial condition $u(0)=u_{0} \in L^{1}(\Omega)$. Let $J_{k}(r)=$ $\int_{0}^{r} T_{k}(s) d s, k>0$. Then

$$
\int_{\Omega} J_{k}(u(t))+\int_{0}^{t}\left\|D T_{k}(u(s))\right\| d s \leq \int_{\Omega} J_{k}\left(u_{0}\right)
$$

for all $t>0$ and all $k>0$.

Proof. Since, almost everywhere on $[0, T],\left(u(t),-u_{t}(t)\right) \in \mathcal{A}$, for almost all $t \in[0, T]$ there exists $z(t) \in X(\Omega)$ with $\|z(t)\|_{\infty} \leq 1$ such that $u_{t}(t)=$ $\operatorname{div}(z(t))$ and

$$
\int_{\Omega}\left(T_{k}(u(t))-w\right) u_{t}(t) \leq \int_{\Omega} z(t) \cdot \nabla w d x-\left\|D T_{k}(u(t))\right\|
$$


for all $w \in W^{1,1}(\Omega) \cap L^{\infty}(\Omega)$, and all $k>0$. Now set $w=0$ in (56) to get

$$
\frac{d}{d t} \int_{\Omega} J_{k}(u(t))+\left\|D T_{k}(u(t))\right\| \leq 0 .
$$

Integrating this expression we obtain (55).

Lemma 6. Let $u, v \in L^{1}(\Omega), u \in B V(\Omega), z \in X(\Omega)$, with $\|z\|_{\infty} \leq 1$ and $v=-\operatorname{div}(z)$. Suppose that

$$
\int_{\Omega}(w-u) v \leq \int_{\Omega} z \cdot \nabla w d x-\|D u\| \quad \forall w \in W^{1,1}(\Omega) \cap L^{\infty}(\Omega) .
$$

Then

$$
\int_{\Omega}\left(w-T_{k}(u)\right) v \leq \int_{\Omega} z \cdot \nabla w d x-\left\|D T_{k}(u)\right\|
$$

$\forall w \in W^{1,1}(\Omega) \cap L^{\infty}(\Omega), \forall k>0$.

Proof. As in Lemma 4, we observe that we may use test functions $w \in$ $B V(\Omega) \cap L^{\infty}(\Omega)$ in (57). If we set $w=u$ in (57) we have that

$$
\int_{\Omega}(z, D u)=\|D u\|
$$

Since

$$
\int_{\Omega}\left(z, D T_{k}(u)\right) \leq\left\|D T_{k}(u)\right\|, \quad \int_{\Omega}\left(z, D G_{k}(u)\right) \leq\left\|D G_{k}(u)\right\|,
$$

and

$\|D u\|=\int_{\Omega}(z, D u)=\int_{\Omega}\left(z, D T_{k}(u)+D G_{k}(u)\right) \leq\left\|D T_{k}(u)\right\|+\left\|D G_{k}(u)\right\|=\|D u\|$

we obtain

$$
\int_{\Omega}\left(z, D T_{k}(u)\right)=\left\|D T_{k}(u)\right\|, \quad \int_{\Omega}\left(z, D G_{k}(u)\right)=\left\|D G_{k}(u)\right\| .
$$

Now, set $w=\varphi+G_{k}(u), \varphi \in W^{1,1}(\Omega) \cap L^{\infty}(\Omega)$, in (57) to obtain

$$
\int_{\Omega}\left(\varphi-T_{k}(u)\right) v \leq \int_{\Omega} z \cdot \nabla \varphi+\int_{\Omega}\left(z, D G_{k}(u)\right)-\|D u\|=\int_{\Omega} z \cdot \nabla \varphi-\left\|D T_{k}(u)\right\| .
$$

We shall need the following lemma, whose proof is straightforward. 
Lemma 7. Let $u \in C\left([0, T], L^{1}(\Omega)\right) \cap W_{l o c}^{1,1}\left(0, T ; L^{1}(\Omega)\right), z \in L^{\infty}((0, T) \times \Omega)$ with $\|z\|_{\infty} \leq 1$ and such that $u_{t}=\operatorname{div}(z)$ in $\mathcal{D}^{\prime}((0, T) \times \Omega)$. Then for almost all $t \in[0, T]$

$$
u_{t}(t)=\operatorname{div}(z(t)) \quad \text { in } \mathcal{D}^{\prime}(\Omega) .
$$

Proof of Theorem 1. Let $u \in C\left([0, T], L^{1}(\Omega)\right) \cap W_{l o c}^{1,1}\left(0, T ; L^{1}(\Omega)\right)$ be the strong solution of (15). Let us assume that $u_{0} \in L^{\infty}(\Omega) \cap D(\mathcal{A})$. By the complete accretivity of $\mathcal{A}$ we know that $\|u(t)\|_{\infty} \leq\left\|u_{0}\right\|_{\infty}$, and taking $k>$ $\left\|u_{0}\right\|_{\infty}$ we conclude by using Lemma 5 that $u \in L^{1}(0, T ; B V(\Omega))$. Since $u(t)$ is a strong solution of (15), the set $K$ consisting of those values of $t \in[0, T]$ for which either $u$ is not differentiable at $t$, or $t$ is not a Lebesgue point for $u^{\prime}$, or $u^{\prime}+\mathcal{A} u \not \supset 0$, is a null subset of $[0, T]$. Then, since $u^{\prime} \in L^{1}\left(0, T ; L^{1}(\Omega)\right)$, Proposition 1.5 in [8] guarantees us that for each $\epsilon>0$, there exists a partition $0=t_{0}<t_{1}<\cdots<t_{n-1} \leq T<t_{n}$ with the properties $t_{k} \notin K$, $t_{k}-t_{k-1}<\epsilon$, for $k=1, \ldots, n$, and

$$
\sum_{k=1}^{n} \int_{t_{k-1}}^{t_{k}}\left\|u^{\prime}(s)-u^{\prime}\left(t_{k}\right)\right\| d s<\epsilon .
$$

If one defines $u_{\epsilon}$ as $u_{\epsilon}(0)=u_{0}, u_{\epsilon}(t)=u\left(t_{k}\right)$ on $\left(t_{k-1}, t_{k}\right], k=1, \ldots, n$, then $u_{\epsilon} \rightarrow u$ in $C\left(0, T ; L^{1}(\Omega)\right)$.

Since $\left(u\left(t_{k}\right),-u^{\prime}\left(t_{k}\right)\right) \in \mathcal{A}$, there exists $z_{k} \in X(\Omega)$, with $u^{\prime}\left(t_{k}\right)=\operatorname{div}\left(z_{k}\right)$ in $\mathcal{D}^{\prime}(\Omega)$ such that

$$
\int_{\Omega}\left(u\left(t_{k}\right)-w\right) u^{\prime}\left(t_{k}\right) \leq \int_{\Omega} z_{k} \cdot \nabla w-\left\|D u\left(t_{k}\right)\right\|
$$

for all $w \in W^{1,1}(\Omega) \cap L^{\infty}(\Omega)$. Thus, if we set $z_{\epsilon}(t)=z_{k}$ and $v_{\epsilon}(t)=u^{\prime}\left(t_{k}\right)$ on $\left(t_{k-1}, t_{k}\right], k=1, \ldots, n$, we get

$$
\int_{0}^{T} \int_{\Omega}\left(u_{\epsilon}-w\right) v_{\epsilon} \varphi \leq \int_{0}^{T}\left\{\int_{\Omega} z_{\epsilon} \cdot \nabla w-\left\|D u_{\epsilon}(t)\right\|\right\} \varphi(t)
$$

for all $w \in W^{1,1}(\Omega) \cap L^{\infty}(\Omega)$ and all $\varphi \in C_{0}^{1}(0, T), \varphi \geq 0$. Now, letting $\epsilon \rightarrow$ $0^{+}$, and applying the Vitali convergence theorem, it follows that there exists $z \in L^{\infty}((0, T) \times \Omega)$ with $\|z\|_{\infty} \leq 1$ such that $u_{t}=\operatorname{div}(z)$ in $\mathcal{D}^{\prime}((0, T) \times \Omega)$ and

$$
\int_{0}^{T} \int_{\Omega}(u-w) u_{t} \varphi \leq \int_{0}^{T}\left\{\int_{\Omega} z \cdot \nabla w-\|D u(t)\|\right\} \varphi(t)
$$


for all $w \in W^{1,1}(\Omega) \cap L^{\infty}(\Omega)$ and all $\varphi \in C_{0}^{1}(0, T), \varphi \geq 0$. Since $(u-w) u_{t} \in$ $L_{\text {loc }}^{1}\left(0, T ; L^{1}(\Omega)\right), \int_{\Omega} z \cdot \nabla w-\|D u(t)\| \in L^{1}(0, T)$ it follows that

$$
\int_{\Omega}(u(t)-w) u_{t}(t) \leq \int_{\Omega} z(t) \cdot \nabla w-\|D u(t)\|
$$

for every $w \in W^{1,1}(\Omega) \cap L^{\infty}(\Omega)$ and almost everywhere on $[0, T]$. Now, using Lemmas 6 and 7 we obtain that

$$
\int_{\Omega}\left(T_{k}(u(t))-w\right) u_{t}(t) \leq \int_{\Omega} z(t) \cdot \nabla w-\left\|D T_{k}(u(t))\right\|
$$

for every $w \in W^{1,1}(\Omega) \cap L^{\infty}(\Omega)$ and almost everywhere on $[0, T]$. We have shown that $u(t)$ is a weak solution of $(1)$.

Now, let $u_{0} \in L^{1}(\Omega)$ and let $u_{0 n} \in L^{\infty}(\Omega) \cap D(\mathcal{A})$. Let $u_{n}, u$ be the strong solutions of (15) with initial data $u_{0 n}, u_{0}$, respectively. We know that $u_{n}$ converges to $u$ in $C\left([0, T], L^{1}(\Omega)\right)$ and $u \in C\left([0, T], L^{1}(\Omega)\right) \cap W_{l o c}^{1,1}\left(0, T ; L^{1}(\Omega)\right)$. By Lemma 5, we have that

$$
\int_{0}^{T}\left\|D T_{k}\left(u_{n}(s)\right)\right\| d s \leq \int_{\Omega} J_{k}\left(u_{0 n}\right)
$$

for all $T>0$ and all $k>0$. It follows that $T_{k}(u) \in L^{1}(0, T ; B V(\Omega))$ for all $k>0$. By the previous paragraph, there exist $z_{n} \in L^{\infty}((0, T) \times \Omega)$ with $\left\|z_{n}\right\|_{\infty} \leq 1$ such that $u_{n t}=\operatorname{div}\left(z_{n}\right)$ in $\mathcal{D}^{\prime}((0, T) \times \Omega)$ and

$$
\int_{0}^{T} \int_{\Omega}\left(T_{k}\left(u_{n}\right)-w\right) u_{n t} \varphi \leq \int_{0}^{T} \int_{\Omega} z_{n} \cdot \nabla w \varphi-\int_{0}^{T}\left\|D T_{k}\left(u_{n}(t)\right)\right\| \varphi(t)
$$

for all $w \in W^{1,1}(\Omega) \cap L^{\infty}(\Omega)$, all $\varphi \in C_{0}^{1}(0, T), \varphi \geq 0$ and all $k>0$. Write the previous expression in the form

$$
\int_{0}^{T} \int_{\Omega}\left(u_{n} w-J_{k}\left(u_{n}\right)\right) \varphi^{\prime} \leq \int_{0}^{T} \int_{\Omega} z_{n} \cdot \nabla w \varphi-\int_{0}^{T}\left\|D T_{k}\left(u_{n}(t)\right)\right\| \varphi(t)
$$

for all $w \in W^{1,1}(\Omega) \cap L^{\infty}(\Omega)$, all $\varphi \in C_{0}^{1}(0, T), \varphi \geq 0$ and all $k>0$. Modulo a subsequence, we may assume that $z_{n} \rightarrow z$ in the weak* topology of $L^{\infty}((0, T) \times \Omega)$. Now letting $n \rightarrow \infty$ in $(62)$ we obtain

$$
\int_{0}^{T} \int_{\Omega}\left(u w-J_{k}(u)\right) \varphi^{\prime} \leq \int_{0}^{T} \int_{\Omega} z \cdot \nabla w \varphi-\int_{0}^{T}\left\|D T_{k}(u(t))\right\| \varphi(t)
$$


for all $w \in W^{1,1}(\Omega) \cap L^{\infty}(\Omega)$, all $\varphi \in C_{0}^{1}(0, T), \varphi \geq 0$ and all $k>0$. Integrating by parts with respect to $t$ in the left-hand side of the above expression we obtain

$$
\int_{0}^{T} \int_{\Omega}\left(T_{k}(u)-w\right) u_{t} \varphi \leq \int_{0}^{T}\left\{\int_{\Omega} z \cdot \nabla w-\left\|D T_{k}(u(t))\right\|\right\} \varphi(t)
$$

for all $w \in W^{1,1}(\Omega) \cap L^{\infty}(\Omega)$, all $\varphi \in C_{0}^{1}(0, T), \varphi \geq 0$ and all $k>0$. Since $\left(T_{k}(u)-w\right) u_{t} \in L_{l o c}^{1}\left(0, T ; L^{1}(\Omega)\right), \int_{\Omega} z \cdot \nabla w-\left\|D T_{k}(u(t))\right\| \in L^{1}(0, T)$ it follows that

$$
\int_{\Omega}\left(T_{k}(u(t))-w\right) u_{t}(t) \leq \int_{\Omega} z(t) \cdot \nabla w-\left\|D T_{k}(u(t))\right\|
$$

for all $w \in W^{1,1}(\Omega) \cap L^{\infty}(\Omega)$ and all $k>0$, almost everywhere on $[0, T]$. Finally observe that $u_{t}=\operatorname{div}(z)$ in $\mathcal{D}^{\prime}((0, T) \times \Omega)$ and $\|z\|_{\infty} \leq 1$. We conclude that $u$ is a weak solution of (1).

For further reference, let us observe that, according to Lemma 4, we also have

$$
\int_{\Omega}\left(T_{k}(u(t))-w\right) u_{t}(t)=\int_{\Omega}(z(t), D w)-\left\|D T_{k}(u(t))\right\|
$$

for all $w \in B V(\Omega) \cap L^{\infty}(\Omega)$ and all $k>0$, almost everywhere on $[0, T]$.

Let us finally observe that a weak solution of (1) is a strong solution of (15). Let $u$ be a weak solution of $(1)$ in $(0, T) \times \Omega$. Then $u \in C\left([0, T], L^{1}(\Omega)\right) \cap$ $W_{l o c}^{1,1}\left((0, T), L^{1}(\Omega)\right), T_{k}(u) \in L^{1}([0, T], B V(\Omega))$ for all $k>0$ and there exists $z \in L^{\infty}((0, T) \times \Omega)$ with $\|z\|_{\infty} \leq 1, u_{t}=\operatorname{div}(z)$ in $\mathcal{D}^{\prime}((0, T) \times \Omega)$ such that

$$
\int_{\Omega}\left(T_{k}(u(t))-w\right) u_{t}(t) \leq \int_{\Omega} z(t) \cdot \nabla w-\left\|D T_{k}(u(t))\right\|
$$

for every $w \in W^{1,1}(\Omega) \cap L^{\infty}(\Omega)$ and almost everywhere on $[0, T]$. By Lemma 7 we have that almost everywhere on $[0, T], u_{t}(t)=\operatorname{div}(z(t))$. Hence almost everywhere on $[0, T], z(t) \in X(\Omega)$ and, since (64) holds also almost everywhere on $[0, T]$, we have that almost everywhere on $[0, T]\left(u(t),-u_{t}(t)\right) \in \mathcal{A}$, i.e.,

$$
u^{\prime}(t)+\mathcal{A} u(t) \ni 0 \quad \text { a.e. on }[0, T] .
$$

Therefore $u$ is a strong solution of (15). The uniqueness of weak solutions of (1) follows as a consequence of the uniqueness of strong solutions of (15). The comparison estimates (7) follow from the complete accretivity of $\mathcal{A}$. 
4. Asymptotic behaviour of solutions. In this section we establish that the mild solutions of problem (1) stabilize as $t \rightarrow 0$ by converging to a constant function. In order to prove the stabilization theorem we need the orbits to be relatively compact.

Lemma 8. Let $(S(t))_{t \geq 0}$ be the semigroup generated by $\mathcal{A}$. Then, for every $u_{0} \in L^{1}(\Omega)$ the orbit $\gamma\left(u_{0}\right)=\left\{S(t) u_{0}: t \geq 0\right\}$ is a relatively compact subset of $L^{1}(\Omega)$.

Proof. Let $J_{\lambda}$ be the resolvent of $\mathcal{A}$. Then, $J_{\lambda}(B)$ is a relatively compact subset of $L^{1}(\Omega)$ if $B$ is a bounded subset of $L^{\infty}(\Omega)$. In fact, let $B$ be a bounded subset of $L^{\infty}(\Omega)$. Take $\left\{f_{n}\right\}_{n=1}^{\infty} \subseteq B$ and let $u_{n}:=J_{\lambda} f_{n}$. Set $M:=\sup _{n \in \mathbb{N}}\left\|f_{n}\right\|_{\infty}<\infty$. Since $\mathcal{A}$ is m-completely accretive, $\left\|u_{n}\right\|_{\infty} \leq M$ for every $n \in \mathbb{N}$. Moreover, since $\left(u_{n}, \frac{1}{\lambda}\left(f_{n}-u_{n}\right)\right) \in \mathcal{A}$, by Lemma 4 ,

$$
\left\|D u_{n}\right\|=\int_{\Omega} \frac{1}{\lambda}\left(f_{n}-u_{n}\right) u_{n} \leq \frac{2}{\lambda} M^{2} \quad \text { for all } n \in \mathbb{N} .
$$

Thus, $\left\{u_{n}: n \in \mathbb{N}\right\}$ is a bounded sequence in $B V(\Omega)$, and by [24, Corollary 5.3.4] we have that $\left\{u_{n}: n \in \mathbb{N}\right\}$ is a relatively compact subset of $L^{1}(\Omega)$.

Consider first $u_{0} \in \mathcal{D}(\mathcal{A}) \cap L^{\infty}(\Omega)$. Then, since $\left\|S(t) u_{0}\right\|_{\infty} \leq\left\|u_{0}\right\|_{\infty}$ for all $t \geq 0$, we have that $J_{\lambda}\left(\gamma\left(u_{0}\right)\right)$ is a relatively compact subset of $L^{1}(\Omega)$ for all $\lambda>0$. Moreover,

$$
\left\|S(t) u_{0}-J_{\lambda} S(t) u_{0}\right\|_{1} \leq \lambda \inf \left\{\|v\|_{1}: v \in \mathcal{A}\left(u_{0}\right)\right\}
$$

Hence, $\gamma\left(u_{0}\right)$ is relatively compact in $L^{1}(\Omega)$. Finally, since $\mathcal{D}(\mathcal{A}) \cap L^{\infty}(\Omega)$ is dense in $L^{1}(\Omega)$, given $u_{0} \in L^{1}(\Omega)$ and $\epsilon>0$, there exists $v_{0} \in \mathcal{D}(\mathcal{A}) \cap L^{\infty}(\Omega)$ such that $\left\|u_{0}-v_{0}\right\|_{1}<\epsilon$. Thus we have

$$
\sup _{t \geq 0} \inf _{s \geq 0}\left\|S(t) u_{0}-S(s) v_{0}\right\|_{1} \leq \sup _{t \geq 0}\left\|S(t) u_{0}-S(t) v_{0}\right\|_{1} \leq\left\|u_{0}-v_{0}\right\|_{1}<\epsilon .
$$

It follows that $\gamma\left(u_{0}\right)$ is relatively compact in $L^{1}(\Omega)$.

We need the following result about the conservation of mass.

Lemma 9. Let $(S(t))_{t \geq 0}$ be the semigroup generated by $\mathcal{A}$. Then, we have conservation of mass; that is,

$$
\int_{\Omega} S(t) u_{0}=\int_{\Omega} u_{0}, \quad \text { for all } t \geq 0 .
$$


Proof. Given $u_{0} \in L^{1}(\Omega)$, let $u(t)=S(t) u_{0}$. Then, $\left(u(t),-u^{\prime}(t)\right) \in \mathcal{A}$. Hence, taking $w=T_{k}(u(t)) \pm 1$ as test function in (34), we obtain that $\int_{\Omega} u^{\prime}(t)=0$. Consequently, the function $t \mapsto \int_{\Omega} u(t)$ is constant, and the proof concludes.

Theorem 3. Let $(S(t))_{t \geq 0}$ be the semigroup generated by $\mathcal{A}$. Then

$$
\left\|S(t) u_{0}-\overline{u_{0}}\right\|_{1} \rightarrow 0 \text { as } t \rightarrow \infty
$$

where $\overline{u_{0}}=\frac{1}{\mu(\Omega)} \int_{\Omega} u_{0}(x) d x$. Moreover, if $u_{0} \in L^{\infty}(\Omega)$ there exists a constant $C$, independent of $u_{0}$, such that

$$
\left\|S(t) u_{0}-\overline{u_{0}}\right\|_{p} \leq \frac{C\left\|u_{0}\right\|_{2}^{2}}{t} \quad \text { for all } t>0, \quad \text { and } \quad 1 \leq p \leq \frac{N}{N-1} .
$$

Proof. Suppose first that $u_{0} \in L^{\infty}(\Omega)$. Since $\mathcal{A}$ is completely accretive then $\|u(t)\|_{\infty} \leq\left\|u_{0}\right\|_{\infty}$. Using $k>\left\|u_{0}\right\|_{\infty}$ and letting $t \rightarrow \infty$ in (55) we have

$$
\int_{0}^{\infty}\left\|D S(\tau) u_{0}\right\| d \tau \leq \frac{1}{2} \int_{\Omega} u_{0}^{2}
$$

Thus, there exists a sequence $t_{n} \rightarrow \infty$, such that $\left\|D S\left(t_{n}\right) u_{0}\right\| \rightarrow 0$ as $n \rightarrow \infty$. Now by Lemma 8 , there exists a subsequence $\left(t_{n_{k}}\right)$ such that

$$
\lim _{k \rightarrow \infty} S\left(t_{n_{k}}\right) u_{0}=v \in \omega\left(u_{0}\right),
$$

and by the lower semicontinuity of the total variation, it follows that

$$
\|D v\| \leq \liminf _{k \rightarrow \infty}\left\|D S\left(t_{n_{k}}\right) u_{0}\right\|=0
$$

Therefore, $v$ is a constant $K$, and consequently, $S(t) K=K$ for all $t \geq 0$, so as $S(t)$ are contractions we get $\omega\left(u_{0}\right)=\{K\}$ and $\lim _{t \rightarrow \infty} S(t) u_{0}=K$. Now, as a consequence of Lemma $9, K=\overline{u_{0}}$ and the proof for the case $u_{0} \in L^{\infty}(\Omega)$ concludes. Now, from the above we obtain easily the conclusion in the general case $u_{0} \in L^{1}(\Omega)$.

Finally, suppose $u_{0} \in L^{\infty}(\Omega)$. Then, by (65) we have that

$$
\int_{0}^{t}\left\|D S(s) u_{0}\right\| d s \leq \frac{1}{2}\left\|u_{0}\right\|_{2}^{2} \quad \forall t>0
$$


On the other hand, since $\overline{S(s) u_{0}}=\overline{u_{0}}$, by the Poincaré-Wirtinger inequality (see, [24, Theorem 5.11.1]), it follows that

$$
\left\|S(s) u_{0}-\overline{u_{0}}\right\|_{p}=\left\|S(s) u_{0}-\overline{S(s) u_{0}}\right\|_{p} \leq M\left\|D S(s) u_{0}\right\|,
$$

for all $s>0$, and $1 \leq p \leq \frac{N}{N-1}$. Then, (66) and (67) imply that

$$
\int_{0}^{t}\left\|S(s) u_{0}-\overline{u_{0}}\right\|_{p} d s \leq \frac{M}{2}\left\|u_{0}\right\|_{2}^{2} \quad \forall t>0 .
$$

Now, since $\mathcal{A}$ is completely accretive and $V(u)=\left\|u-\overline{u_{0}}\right\|_{p}$ is a Lyapunov functional for the semigroup generated by $\mathcal{A}$. Using (68) we get

$$
t\left\|S(t) u_{0}-\overline{u_{0}}\right\|_{p} \leq \int_{0}^{t}\left\|S(s) u_{0}-\overline{u_{0}}\right\|_{p} d s \leq \frac{M}{2}\left\|u_{0}\right\|_{2}^{2}
$$

concluding the proof.

5. Some qualitative properties. We shall prove that the length of the level curves of the solution is a decreasing function of time, as should be expected. We shall also prove that flat zones which are local maxima (minima) immediately decrease (respectively, increase) with time.

Let $T_{a, b}(r)=\min (\max (a, r), b), a, b, r \in \mathbb{R}, a<b$. Let $\psi: B V(\Omega) \rightarrow$ $[0,+\infty)$ be defined by

$$
\psi(u)=\|D u\|, \quad u \in B V(\Omega) .
$$

Proposition 2. Let $u_{0} \in L^{1}(\Omega)$. Let $u(t, x)$ be the weak solution of (1). Then

$$
\psi\left(T_{a, b}(u(t))\right) \leq \psi\left(T_{a, b}(u(s))\right)
$$

almost everywhere in $s, t \in(0, \infty), t>s$ and all $a<b$.

Proof. Assume first that $u_{0} \in L^{2}(\Omega)$. Let $\delta>0$ and $t, s \geq \delta$ such that $\left(u(t),-u_{t}(t)\right),\left(u(s),-u_{t}(s)\right) \in \mathcal{A}$. Let $a, b \in \mathbb{R}$. Assume that $0 \leq a<b$. We have

$$
\int_{\Omega}\left(T_{b}(u(t))-w\right) u_{t}(t) \leq \int_{\Omega}(z(t), D w)-\left\|D T_{b}(u(t))\right\|
$$


for all $w \in B V(\Omega) \cap L^{\infty}(\Omega)$. Use as test function $w-a+T_{-b, a}(u(t))$. Then

$$
\begin{aligned}
& \int_{\Omega}\left(T_{a, b}(u(t))-w\right) u_{t}(t) \\
& \leq \int_{\Omega}(z(t), D w)+\int_{\Omega}\left(z(t), D T_{-b, a}(u(t))\right)-\left\|D T_{b}(u(t))\right\| \\
& \leq \int_{\Omega}(z(t), D w)-\left\|D T_{a, b}(u(t))\right\|
\end{aligned}
$$

for all $w \in B V(\Omega) \cap L^{\infty}(\Omega)$. Consequently, we have

$$
\int_{\Omega}\left(T_{a, b}(u(t))-w\right) u_{t}(t) \leq \int_{\Omega}(z(t), D w)-\left\|D T_{a, b}(u(t))\right\|
$$

for all $w \in B V(\Omega) \cap L^{\infty}(\Omega)$ and all $a, b \in \mathbb{R}, a<b$. Setting $w=T_{a, b}(u(s))$ in (71) we have

$$
\left\|D T_{a, b}(u(t))\right\|-\left\|D T_{a, b}(u(s))\right\| \leq \int_{\Omega} u_{t}(t)\left(T_{a, b}(u(s))-T_{a, b}(u(t))\right) .
$$

Using estimate (52) we may write

$$
\left\|D T_{a, b}(u(t))\right\|-\left\|D T_{a, b}(u(s))\right\| \leq \frac{2}{\delta}\left\|u_{0}\right\|_{2}\left\|T_{a, b}(u(s))-T_{a, b}(u(t))\right\|_{2} .
$$

Since a similar estimate holds with $s$ and $t$ interchanged, we have

$$
\left|\left\|D T_{a, b}(u(t))\right\|-\left\|D T_{a, b}(u(s))\right\|\right| \leq \frac{2}{\delta}\left\|u_{0}\right\|_{2}\left\|T_{a, b}(u(s))-T_{a, b}(u(t))\right\|_{2} .
$$

Since $u \in W_{l o c}^{1,1}\left((0, T), L^{2}(\Omega)\right)$, i.e, is a locally absolutely continuous function of time, then also $T_{a, b}(u)$ is, and, from (72), we deduce that $\psi\left(T_{a, b}(u)\right)$ is absolutely continuous in $[0, T]$ for all $T>0$. Let $t \in[0, \infty)$ be such that $u, T_{a, b}(u), \psi\left(T_{a, b}(u)\right)$ are differentiable at $t$ and $\left(u(t),-u_{t}(t)\right) \in \mathcal{A}$. Set $w=T_{a, b}(u(t+\epsilon)), w=T_{a, b}(u(t-\epsilon))$ in (71) to obtain

$$
\int_{\Omega}\left(T_{a, b}(u(t))-T_{a, b}(u(t \pm \epsilon))\right) u_{t}(t) \leq \psi\left(T_{a, b}(u(t \pm \epsilon))\right)-\psi\left(T_{a, b}(u(t))\right) .
$$

Letting $\epsilon \rightarrow 0+$ we have

$$
\frac{d}{d t} \psi\left(T_{a, b}(u(t))\right)=-\int_{\Omega} T_{a, b}^{\prime}(u(t)) u_{t}(t)^{2} \leq 0
$$


Hence, $\psi\left(T_{a, b}(u(t))\right)$ is a decreasing function of time. In particular, for all $s<t$ such that $T_{a, b}(u(s)), T_{a, b}(u(t)) \in B V(\Omega)$, hence almost everywhere in $s, t, s<t$, we have

$$
\psi\left(T_{a, b}(u(t))\right) \leq \psi\left(T_{a, b}(u(s))\right) .
$$

In particular, if $u(0) \in L^{2}(\Omega)$ and $T_{a, b}(u(0)) \in B V(\Omega)$ for all $a<b$, then

$$
\psi\left(T_{a, b}(u(t))\right) \leq \psi\left(T_{a, b}(u(0))\right)
$$

almost everywhere in $t \in(0, \infty)$, for all $a<b$.

Now, let $u_{0} \in L^{1}(\Omega)$ and $u(t)$ be the corresponding weak solution of (1). Let $s>0$ be such that $T_{a, b}(u(s)) \in B V(\Omega)$ for all $a<b$ (which is possible by the proof of Lemma 3). Take $s$ as the origin of time. Let $u^{n}(s) \in$ $L^{2}(\Omega) \cap B V(\Omega)$, such that $u^{n}(s) \rightarrow u(s)$ in $L^{1}(\Omega)$ and $\left\|D T_{a, b}\left(u^{n}(s)\right)\right\| \rightarrow$ $\left\|D T_{a, b}(u(s))\right\|$ as $n \rightarrow \infty$, for all $a<b$. Let $u^{n}(t, x)$ be the weak solution of (1) with initial condition at $t=s, u^{n}(s, x)=u^{n}(s)(x)$. Then $u^{n}(t) \rightarrow u(t)$ in $C\left([s, T], L^{1}(\Omega)\right)$ for all $T>s$. Using (74) we have

$$
\psi\left(T_{a, b}\left(u^{n}(t)\right)\right) \leq \psi\left(T_{a, b}\left(u^{n}(s)\right)\right),
$$

for almost all $t \in(s, \infty)$ and all $a<b$. Letting $n \rightarrow \infty$, we get

$$
\psi\left(T_{a, b}(u(t))\right) \leq \psi\left(T_{a, b}(u(s))\right),
$$

for almost all $t \in(s, \infty)$ and all $a<b$. Hence (75) holds for almost all $s, t \in(0, \infty), s<t$, and all $a<b$.

Lemma 10. Let $g(t, \lambda) \in L_{\text {loc }}^{1}((0, \infty) \times \mathbb{R})$. Let $\Delta=\{(s, t) \in(0, \infty) \times$ $(0, \infty): s<t\}$. Suppose that

$$
\int_{a}^{b} g(t, \lambda) d \lambda \leq \int_{a}^{b} g(s, \lambda) d \lambda
$$

almost everywhere in $(s, t) \in \Delta$ and all $a<b$. Then $g(t, \lambda) \leq g(s, \lambda)$ almost everywhere in $(s, t, \lambda) \in \Delta \times \mathbb{R}$.

Proof. Consider the function $G(s, t, \lambda)$ defined in $\Delta \times \mathbb{R}$ by $G(s, t, \lambda)=$ $g(t, \lambda)-g(s, \lambda)$. Let $\left(s_{0}, t_{0}, \lambda_{0}\right)$ be a Lebesgue point of $G$. By assumption we know that for $r>0$ and almost all $(s, t) \in \Delta, \int_{\lambda_{0}-r}^{\lambda_{0}+r} G(s, t, \lambda) d \lambda \leq 0$. Hence,

$$
G\left(s_{0}, t_{0}, \lambda_{0}\right)=\lim _{r \rightarrow 0^{+}} \frac{1}{(2 r)^{3}} \int_{s_{0}-r}^{s_{0}+r} \int_{t_{0}-r}^{t_{0}+r} \int_{\lambda_{0}-r}^{\lambda_{0}+r} G(s, t, \lambda) d s d t d \lambda \leq 0 .
$$

Since almost all points of $\Delta \times \mathbb{R}$ are Lebesgue points of $G$, the lemma follows. 
Corollary 1. Let $u_{0} \in L^{1}(\Omega)$. Let $u(t, x)$ be the weak solution of (1). Then, for almost all $\lambda \in \mathbb{R}$,

$$
\left\|D \chi_{\{u(t)>\lambda\}}\right\| \leq\left\|D \chi_{\{u(s)>\lambda\}}\right\|
$$

a.e. in $s, t \in(0, \infty), t>s>0$.

Proof. Let $g(t, \lambda)=\left\|D \chi_{\{u(t)>\lambda\}}\right\|$. By Proposition 2 and the co-area formula, $g$ satisfies the assumptions of lemma 10. Then the conclusion follows from that lemma.

Note that $\left\|D \chi_{\{u(t)>\lambda\}}\right\|=H^{N-1}\left(\partial^{*}\{u(t)>\lambda\}\right)$, where $H^{N-1}$ is the $(N-1)$-dimensional Hausdorff measure and $\partial^{*}\{u(t)>\lambda\}$ is the reduced boundary of the set $\{x \in \Omega: u(t)>\lambda\}$ (see [24] or [15]).

Next, we prove that flat zones which are local maxima (minima) immediately decrease (respectively, increase) with time. To simplify our presentation, let us take $\Omega$ to be a cube in $\mathbb{R}^{N}$.

Proposition 3. Let $u_{0} \in C(\bar{\Omega}), 0 \leq u_{0} \leq 1$. Suppose that $\left\{x \in \bar{\Omega}: u_{0}(x)=\right.$ $1\}=K \subseteq B \subset \subset \Omega$ for some ball $B$. Let $u$ be the weak solution of (1). Then $u(t, x)<1$, for all $t>0, x \in \Omega$.

We shall use a comparison principle for the Dirichlet problem together with explicit supersolutions to prove Proposition 3.

Let $g \in C^{2}\left(\mathbb{R}^{+}\right), g(r)>0, g^{\prime}(r)<0$, for $r>0$. Let $M>0, M<$ $g(0+)$. Let $U(x)=\min (g(|x|), M), x \in \mathbb{R}^{N}$. Let $r_{0}>0$ be such that $g\left(r_{0}\right)=M$. Then $U(x)=M$ for $|x| \leq r_{0}$ and $U(x)=g(|x|)$ for $|x|>r_{0}$. Let $R>r_{0}, B_{R}=B(0, R)$. Let us construct a function $U(t, x)$ such that $U \in C\left([0, T], B_{R}\right) \cap W^{1,1}\left((0, T), L^{1}\left(B_{R}\right)\right), U \in L^{1}\left([0, T], B V\left(B_{R}\right)\right), U(0, x)=$ $U(x)$ and there exists $Z(t) \in X\left(B_{R}\right)$ with $\|Z(t)\|_{\infty} \leq 1$, such that for $T>0$ small enough and $t \in[0, T]$, we have

$$
U_{t}=\operatorname{div}(Z(t)) \quad \text { in } \mathcal{D}^{\prime}\left(B_{R}\right)
$$

and

$$
\int_{B_{R}} Z(t) \cdot D U(t)=\|D U(t)\| .
$$

Let us observe that if $u(x)=h(|x|)$, where $h \in C^{2}\left(\mathbb{R}^{+}\right), h(r)>0, h^{\prime}(r)<0$, for $r>0$, then $\operatorname{div}\left(\frac{D u}{|D u|}\right)=-\frac{N-1}{|x|}$. We expect the solution $U(t, x)$ to be a radial function. Then if the flat zone of $U(x)$ has to remain flat we should have that

$$
U_{t}=\left\{\begin{array}{lll}
-\lambda & \text { if } & |x|<r(t) \\
-\frac{N-1}{|x|} & \text { if } & |x|>r(t)
\end{array}\right.
$$


for some $\lambda>0$ and some $r(t)>0$ to be determined and such that $r(0)=r_{0}$. Assume that $Z(t)=-\frac{x}{|x|}$ for $|x|>r(t)$. This choice is consistent with (78). Now, in $B(0, r(t)), Z(t)$ must be such that

$$
\operatorname{div}(Z(t))=-\lambda \quad \text { with }\left.Z(t)\right|_{\partial B(0, r(t))}=-\frac{x}{|x|},\|Z(t)\|_{\infty} \leq 1 .
$$

Integrating the previous equation in $B(0, r(t))$, we have

$$
\begin{aligned}
-\lambda \operatorname{meas}(B(0, r(t))) & =\int_{B(0, r(t))} \operatorname{div}(Z(t)) d x=\int_{\partial B(0, r(t))} z \cdot n \\
& =-H^{N-1}(\partial B(0, r(t))),
\end{aligned}
$$

and, therefore, $\lambda=\frac{H^{N-1}(\partial B(0, r(t)))}{\text { meas }(B(0, r(t)))}=\frac{N}{r(t)}$. Then, we may take $Z(t)=-\frac{x}{r(t)}$ when $|x|<r(t)$. Such a choice of $Z(t)$ satisfies (81). We have to choose $r(t)$ such that $U(t, x)$ remains a Lipschitz function, in particular continuous for $|x|=r(t)$. Assume for the time being that $r^{\prime}(t)>0$. Let us observe that

$$
U(t, 0)=U(0)-N \int_{0}^{t} \frac{d s}{r(s)} .
$$

Now, if $x \in B_{R}$ is a point such that $|x|>r(t)$, then

$$
U(t, x)=U(x)+\int_{0}^{t} U_{t}(s, x) d s=U(x)-\frac{t(N-1)}{|x|} .
$$

Since the value $U(t, r(t)-)$ must coincide with the value of $U(t, 0)$ and $U(t, r(t)-)=U(t, r(t)+)$, then

$$
U(0)-N \int_{0}^{t} \frac{d s}{r(s)}=U(r(t)+)-\frac{t(N-1)}{|r(t)|} .
$$

Thus, differentiating the above expression we see that $r(t)$ must satisfy the differential equation

$$
r^{\prime}(t)=-\frac{r(t)}{(N-1) t+g^{\prime}(r(t)) r(t)^{2}} .
$$

We take $r(t)$ to be the solution of (82) such that $r(0)=r_{0}$. Then, the function

$$
U(t, x)=\left\{\begin{array}{lll}
U(0)-N \int_{0}^{t} \frac{d s}{r(s)} & \text { if } & |x|<r(t) \\
U(x)-(N-1) \frac{t}{|x|} & \text { if } & |x|>r(t) .
\end{array}\right.
$$


satisfies (78) for $t>0$ small, $Z(t)$ being given by

$$
Z(t)=\left\{\begin{array}{lll}
-\frac{x}{r(t)} & \text { if } & |x|<r(t) \\
-\frac{x}{|x|} & \text { if } & |x|>r(t) .
\end{array}\right.
$$

Observe that $\|Z(t)\|_{\infty} \leq 1$ and satisfies (79) for $t>0$ small.

Thus, the following result is established.

Proposition 4. There is some $T>0$ such that the function $U$ defined in (83) is a solution of $(78)$ in $\mathcal{D}^{\prime}\left((0, T) \times B_{R}\right)$ satisfying $(79)$ for all $t \in[0, T]$ and such that $U(0, x)=U(x)$. Moreover, $U \in C\left([0, T], B_{R}\right) \cap W^{1,1}((0, T)$, $\left.L^{1}\left(B_{R}\right)\right)$, and $U \in L^{1}\left([0, T], B V\left(B_{R}\right)\right)$.

Proof of Proposition 3. Without loss of generality we may assume that $B=B(0, R)$. Let $U(x)=\min (g(|x|), 1)$ where $g \in C^{2}\left(\mathbb{R}^{+}\right), g(r)>0$, $g^{\prime}(r)<0$, for $r>0$. Assume that $g(0+)>1, g(R)<1$. Assume also that $\sup _{x \in \partial B} u_{0}<g(R)$ and $u_{0}(x) \leq U(x)$ for $x \in B$. We observe that $u \in C([0, T] \times \bar{\Omega})$, for all $T>0$, when $u_{0} \in C(\bar{\Omega})$. For that, assume that $u_{0} \in W^{1, \infty}(\Omega)$. Then $u(t) \in W^{1, \infty}(\Omega)$ for all $t>0$. Indeed, let $u_{0} \in W^{1, \infty}(\Omega)$. We know that the solution $u_{\epsilon}$ of

$$
\begin{array}{ll}
\frac{\partial u}{\partial t}=\operatorname{div}\left(\frac{D u}{\left(\epsilon^{2}+|D u|^{2}\right)^{1 / 2}}\right)+\epsilon \Delta u & \text { in } Q=(0, \infty) \times \Omega \\
\frac{\partial u}{\partial \eta}=0 \quad & \text { in } S=(0, \infty) \times \partial \Omega \\
u(0, x)=u_{0}(x) \quad \text { for } x \in \Omega
\end{array}
$$

converges in $L^{2}(\Omega)$ to the weak solution of (1) (see [19]). If we extend $u_{0}(x)$ to $\mathbb{R}^{N}$ by reflection as an even function and then by periodicity we obtain a function $\bar{u}_{0}(x)$ defined in $\mathbb{R}^{N}$. Then if $\bar{u}_{\epsilon}(t, x)$ denotes the solution of (85) in $(0, \infty) \times \mathbb{R}^{N}$ with initial condition $\bar{u}_{0}(x)$ then $u_{\epsilon}(t, x)=\bar{u}_{\epsilon}(t, x)$ for $x \in \Omega$, $t>0$. Now, using the Bernstein method as in [1], it is easy to see that

$$
\|\nabla u(t)\|_{\infty} \leq\left\|\nabla u_{0}\right\|_{\infty}, \quad \text { for all } t>0 .
$$

Then it follows that $u(t) \in W^{1, \infty}(\Omega)$ for all $t>0$ with Lipschitz constant $\left\|\nabla u_{0}\right\|_{\infty}$. On the other hand, since $\mathcal{A}$ is $m$-completely accretive in $L^{1}(\Omega)$, $\mathcal{A} \cap\left(L^{\infty}(\Omega) \times L^{\infty}(\Omega)\right)$ generates a strongly continuous semigroup in $L^{\infty}(\Omega)$. Consequently, $u$ is a continuous function in $(t, x)$. Now, let $u_{0} \in C(\bar{\Omega})$, and $u(t, x)$ be the corresponding weak solution of $(1)$. Let $u_{0 n} \in W^{1, \infty}(\Omega)$ be 
such that $u_{0 n} \rightarrow u_{0}$ in $C(\bar{\Omega})$ as $n \rightarrow \infty$. If $u_{n}$ is the weak solution of (1) with initial datum $u_{0 n}$, then $u_{n} \in C([0, T] \times \bar{\Omega})$ converges to $u$ in $C\left([0, T], L^{\infty}(\Omega)\right)$ as $n \rightarrow \infty$. Therefore, $u \in C([0, T] \times \bar{\Omega})$.

Since $u(t, x)$ is a continuous function of $(t, x)$, for some $T>0$, we have that $u(t, x) \leq U(t, x)$, for all $t \in[0, T], x \in \partial B$. Let us prove that $u(t, x) \leq$ $U(t, x)$, for all $t \in[0, T], x \in B$, where $U(t, x)$ is the solution constructed in Proposition 4. Since $u$ is a strong solution of (15), there exists $z(t) \in X(\Omega)$ such that

$$
-\int_{\Omega}(w-u(t)) u_{t}(t)=\int_{\Omega}(z(t), D w)-\|D u(t)\|
$$

for all $w \in B V(\Omega) \cap L^{\infty}(\Omega)$. Taking $\tilde{w}=u(t)+w \varphi-u(t) \varphi$ in (88), it follows that

$$
\begin{aligned}
& -\int_{\Omega}(w-u(t)) \varphi u_{t}(t) \\
& =\int_{\Omega}(z(t), D w) \varphi-\int_{\Omega}(z(t), D u(t)) \varphi+\int_{\Omega}(z(t), D \varphi)(w-u(t))
\end{aligned}
$$

for all $w \in B V(\Omega) \cap L^{\infty}(\Omega)$ and $\varphi \in \mathcal{D}(\Omega)$. Let us take now $\varphi_{n} \in \mathcal{D}(\Omega)$ such that $\varphi_{n} \rightarrow \chi_{B}$ and $\int_{\Omega}\left\|\nabla \varphi_{n}\right\| \rightarrow\left\|D \chi_{B}\right\|$. Then

$$
\begin{gathered}
-\int_{\Omega}(w-u(t)) \varphi_{n} u_{t}(t) \\
=\int_{\Omega}(z(t), D w) \varphi_{n}-\int_{\Omega}(z(t), D u(t)) \varphi_{n}+\int_{\Omega}\left(z(t), D \varphi_{n}\right)(w-u(t)) .
\end{gathered}
$$

By Theorem 4.2 in [5], we have that

$$
\begin{aligned}
& \lim _{n \rightarrow \infty} \int_{\Omega}\left(z(t), D \varphi_{n}\right)(w-u(t))=\int_{\Omega}\left(z(t), D \chi_{B}\right)(w-u(t)) \\
& =-\int_{\partial B}(z(t) \cdot \eta)(w-u(t))
\end{aligned}
$$

for all $w \in C(\Omega) \cap L^{\infty}(\Omega)$. Letting $n \rightarrow \infty$ in (89) we get

$$
\begin{aligned}
-\int_{B}(w-u(t)) u_{t}(t) & =\int_{B}(z(t), D w)-\int_{B}(z(t), D u(t)) \\
& -\int_{\partial B}(z(t) \cdot \eta)(w-u(t))
\end{aligned}
$$


for all $w \in W^{1,1}(\Omega) \cap C(\Omega) \cap L^{\infty}(\Omega)$. On the other hand, by Proposition 4 we have that

$$
\begin{gathered}
\int_{B}(w-U(t)) U_{t}(t)=\int_{B}(w-U(t)) \operatorname{div} Z(t) \\
=-\int_{B}\left(Z(t), D(w-U(t))+\int_{\partial B}(Z(t) \cdot \eta)(w-U(t)) .\right.
\end{gathered}
$$

Taking $w=u(t)+p(u(t)-U(t))$ in $(90)$ and $w=U(t)-p(u(t)-U(t))$ in (91) and adding these equalities we obtain

$$
\begin{aligned}
\int_{B} p(u(t)-U(t))\left(u_{t}(t)-U_{t}(t)\right) & =-\int_{B}(z(t)-Z(t), D p(u(t)-U(t))) \\
& +\int_{\partial B}(z(t)-Z(t)) \cdot \eta p(u(t)-U(t))
\end{aligned}
$$

Working as in the proof of Theorem 2, we have that

$$
-\int_{B}((z(t)-Z(t)), D p(u(t)-U(t))) \leq 0
$$

Since $u(t) \leq U(t)$ on $\partial B, t \in[0, T]$, if we take $p$ converging to $\operatorname{sign}^{+}$, it follows that

$$
\frac{d}{d t} \int_{B}(u(t)-U(t))^{+} \leq 0 \quad \text { for } t \in[0, T]
$$

Since $u_{0}(x) \leq U(x)$, it follows that $u(t, x) \leq U(t, x)$ for $t \in[0, T], x \in B$. Moreover, we may take $T>0$ small enough so that $u(t, x)<1$ for all $t \in[0, T], x \in \Omega \backslash B$. Thus, we have that $u(t, x)<1$ for all $t \in[0, T], x \in \Omega$. Now comparing $u(t, x)$ with constant functions we see that $u(t, x)<1$ for all $t>0, x \in \Omega$.

Remark. We can also compute explicitly the evolution of the characteristic function of a ball $B(p, r)$ when $\Omega$ is a ball centered at $p$. To fix ideas, let $p=0, \Omega=B(0, R)$ and $u_{0}(x)=k \chi_{B(0, r)}$, where $0<r<R$ and $k>0$. We look for a solution of (1) of the form $u(t, x)=\alpha(t) \chi_{B(0, r)}+\beta(t) \chi_{B(0, R) \backslash B(0, r)}$ on some time interval $(0, T)$ defined by the inequalities $\alpha(t)>\beta(t)$ for all $t \in(0, T)$, and $\alpha(0)=k, \beta(0)=0$. Then, we shall look for some 
$z \in L^{\infty}((0, T) \times B(0, R))$ with $\|z\|_{\infty} \leq 1$, such that

$$
\begin{array}{lc}
\alpha^{\prime}(t)=\operatorname{div}(z) & \text { in }(0, T) \times B(0, r) \\
z=-\frac{x}{|x|} & \text { on }(0, T) \times \partial B(0, r), \\
\beta^{\prime}(t)=\operatorname{div}(z) & \text { in }(0, T) \times(B(0, R) \backslash B(0, r)) \\
z=-\frac{x}{|x|} & \text { on }(0, T) \times \partial B(0, r) \\
z \cdot n=0 & \text { on }(0, T) \times \partial B(0, R) \\
\int_{B(0, R)} z \cdot D u=\int_{B(0, R)}|D u| & \text { for all } t \in(0, T) .
\end{array}
$$

Integrating equation (92) in $B(0, r)$, we obtain

$$
\alpha^{\prime}(t)|B(0, r)|=\int_{B(0, r)} \operatorname{div}(z) d x=\int_{\partial B(0, r)} z \cdot n=-H^{N-1}(\partial B(0, r)) .
$$

Thus $\alpha^{\prime}(t)=-\frac{N}{r}$, and, therefore, $\alpha(t)=k-\frac{N}{r} t$. In this case we take $z=-\frac{x}{r}$ and (92) holds. Similarly, we deduce that $\beta^{\prime}(t)=\mu:=N \frac{r^{N-1}}{R^{N}-r^{N}}$; hence, $\beta(t)=N \frac{r^{N-1}}{R^{N}-r^{N}} t$. Our first observation is that $T$ is given by

$$
T\left(\frac{N}{r}+N \frac{r^{N-1}}{R^{N}-r^{N}}\right)=k .
$$

To construct $z$ in $(0, T) \times(B(0, R) \backslash B(0, r))$ we shall look for $z$ of the form $z=\rho(|x|) \frac{x}{|x|}$ such that $\operatorname{div}(z)=\beta^{\prime}(t), \rho(r)=-1, \rho(R)=0$. Since

$$
\operatorname{div}(z)=\nabla \rho(|x|) \cdot \frac{x}{|x|}+\rho(|x|) \operatorname{div}\left(\frac{x}{|x|}\right)=\rho^{\prime}(|x|)+\rho \frac{N-1}{|x|},
$$

we must have

$$
\rho^{\prime}(s)+\rho \frac{N-1}{s}=N \frac{r^{N-1}}{R^{N}-r^{N}} \quad s \in(r, R) .
$$

The solution of (96) such that $\rho(R)=0$ is $\rho(s)=\frac{\mu s}{N}-\frac{\mu R^{N}}{N s^{N-1}}$, which also satisfies $\rho(r)=-1$. Thus, in $B(0, R) \backslash B(0, r), z=\frac{\mu x}{N}-\frac{\mu R^{N} x}{N|x|^{N}}$. It is easy to check that (94) holds. Thus

$$
u(t, x)=\left(k-\frac{N}{r} t\right) \chi_{B(0, r)}+\frac{N r^{N-1}}{R^{N}-r^{N}} t \chi_{B(0, R) \backslash B(0, r)}
$$


in $(0, T) \times B(0, R)$ where $T$ is given by (95). On the other hand, we take

$$
u(t, x)=\left(k-\frac{N}{r} T\right) \chi_{B(0, R)}=\frac{N r^{N-1}}{R^{N}-r^{N}} T \chi_{B(0, R)},
$$

and $z(t, x)=0$ in $(T, \infty) \times B(0, R)$. It is easy to check that $u(t, x)$ is the solution of $(1)$ in $(0, \infty) \times B(0, R)$ with initial datum $u_{0}(x)$. Exact solutions for the minimization problem with constraints (2) in $R^{N}$ with $N=1,2,3$ have been given in $([21])$.

6. Numerical experiments. Minimizing total variation submitted to constraints is one of the most successful techniques for image restoration. It was introduced by L. Rudin and S. Osher in [17], [18]. The first numerical schemes were explicit ([17], [18]). Later, it became evident that one needed faster and more accurate numerical schemes. This was already noticed by S. Osher and L. Rudin in unpublished work. Many papers report the efforts in this sense [11], [12], [10], [22], and [23], to mention a few of them.

Our purpose is not to introduce a new numerical scheme but to display some phenomena which may be useful to understand the qualitative behavior of equation (1). A detailed discussion of numerical schemes to minimize total variation in image restoration can be seen in the references given above.

To discretize equation (1) we shall use an implicit Euler discretization in time,

$$
\frac{u^{n+1}-u^{n}}{\Delta t}=\operatorname{div}\left(\frac{D u^{n+1}}{\sqrt{\epsilon^{2}+\left|D u^{n+1}\right|^{2}}}\right), \quad u^{0}=u_{0},
$$

with $\epsilon>0$ and small. This is a quasilinear elliptic equation, which we solve using fixed-point iteration. Writing $w$ instead of $u^{n+1}, f$ instead of $u^{n}$, we have to solve at each time step an equation like

$$
w-\Delta t \operatorname{div}\left(\frac{D w}{\sqrt{\epsilon^{2}+|D w|^{2}}}\right)=f .
$$

Now, let $A(v, w)=-\operatorname{div}\left(\frac{D w}{\sqrt{\epsilon^{2}+|D v|^{2}}}\right)$. Then, to solve (98) we use the fixedpoint iteration

$$
w^{k+1}+\Delta t A\left(w^{k}, w^{k+1}\right)=f .
$$

We shall stop this iterative process when $\left\|f-w^{k+1}-\Delta t A\left(w^{k}, w^{k+1}\right)\right\| \leq \rho$, for some small $\rho>0$. For each $k$, the space discretization of (99) is a linear system which we solve using the Gauss-Seidel method. 
We performed some numerical experiments to check the evolution of flat zones and the asymptotic behavior of equation (1). The main conclusion being that implicit schemes capture the essential features of the evolution according to (1).

In Figure 1 (Figures 1.1 to 1.6), we see the evolution of a truncated cone. Figures 1.1 and 1.2 display the original image and its corresponding sectional view at $x_{1}=64, x_{2} \in[0,127]$. Figures 1.3 to 1.6 display the same sectional view of the solution after 100, 200, 600 and 1000 time steps, respectively. We shall always use $\Delta t=0.4, \epsilon=0.1$, in our numerical experiments. We see that the level of the flat zone is strictly decreasing and remains a flat zone while its radius increases with time.

In Figure 2, we display the evolution of a truncated cone whose upper level set contains concavities. Figure 2.1 displays the original image. Figure 2.2 displays the solution after 100 time steps. Figures 2.3 and 2.4 display the level lines of each of the images in Figure 2.1 and 2.2, respectively, taken at multiples of 10, i.e., the boundaries of the sets $\left\{\left(x_{1}, x_{2}\right): u\left(t, x_{1}, x_{2}\right) \geq 10 k\right\}$, $k=0,1, \ldots, 25$. Figures 2.5 and 2.6 display the sectional views of the original image corresponding to $x_{1}=64, x_{2} \in[0,127]$ (left) and to $x_{2}=64$, $x_{1} \in[0,127]$ (right). Figures 2.7 to 2.10 display the same sectional views of the solution after 10 and 25 time steps, respectively. We also see how the level of the flat zone is strictly decreasing and remains a flat zone. The initial condition was constructed as follows. Let $C$ be the set in white in Figure 2.1. We computed $d\left(\left(x_{1}, x_{2}\right), C\right)=$ the distance of $\left(x_{1}, x_{2}\right) \in[0,127] \times[0,127]$ to $C$. Then we defined $u_{0}\left(x_{1}, x_{2}\right)=250-d\left(\left(x_{1}, x_{2}\right), C\right)$.

In Figure 3 we display the evolution of a Gaussian-like function. A flat zone is immediately created at the maximum. We also see how the radius of the flat zone increases while its level decreases with time. Figures 3.1 and 3.2 are the original image and its corresponding sectional view at $x_{1}=64, x_{2} \in[0,127]$. Figures 3.3 to 3.5 display the same sectional view of the solution after 50, 100 and 1000 time steps, respectively.

In Figure 4 we check the asymptotic behavior of the solution as $t \rightarrow \infty$. Figures 4.1 and 4.2 are the original image and the solution after 3000 time steps. Figures 4.3 and 4.4 display the sectional view of Figures 4.1 and 4.2 corresponding to $x_{1}=20, x_{2} \in[0,39]$. We can see that the mass is conserved during evolution.

Acknowledgments. The first and fourth authors have been partially supported by the Spanish DGICYT, Project PB94-096. The second and third 
authors were partially supported by the Spanish DGICYT, Project PB941174 and by TMR European Project Viscosity Solutions and their Applications.

\section{REFERENCES}

[1] L. Alvarez, P.L. Lions, and J.M. Morel, Image selective smoothing and edge detection by nonlinear diffusion, SIAM J. Numer. Anal. 29 (1992), 845-866.

[2] L. Alvarez, F. Guichard, P. L. Lions, and J. M. Morel, Axioms and fundamental equations of image processing, Arch. Rational Mechanics and Anal., 16, IX (1993), 200-257.

[3] L. Ambrosio, N. Fusco and D. Pallara, "Functions of Bounded Variation and Free Discontinuity Problems," forthcoming book.

[4] F. Andreu, J.M. Mazón, S. Segura and J. Toledo, Quasilinear elliptic and parabolic equations in $L^{1}$ with nonlinear boundary conditions, Adv. in Math. Sci. and Appl., 7 (1977), 183-213.

[5] G. Anzellotti, Pairings Between Measures and Bounded Functions and Compensated Compactness, Ann. di Matematica Pura ed Appl., IV (135) (1993), 293-318.

[6] Ph. Benilan and M.G. Crandall, "Regularizing Effects in Homogeneous Equations," in Contributions to Analysis and Geometry, D.N. Clark et al. editors, John Hopkins University Press, 1981, 23-39.

[7] Ph. Benilan and M.G. Crandall, Completely Accretive Operators, in "Semigroups Theory and Evolution Equations," Ph. Clement et al. editors, Marcel Dekker, 1991, $41-76$.

[8] Ph. Benilan, M.G. Crandall and A. Pazy "Evolution Equations Governed by Accretive Operators," forthcoming.

[9] H. Brezis, "Operateurs Maximaux Monotones," North Holland, Amsterdam, 1973.

[10] A. Chambolle and P.L. Lions, Image Recovery via Total Variation Minimization and Related Problems, preprint, 1995.

[11] T.F. Chan, G.H. Golub and P. Mulet, A nonlinear primal-dual method for total variation based image restoration, SIAM J. Sci. Computing, to appear.

[12] T.F. Chan, G.H. Golub and P. Mulet, Total Variation Image Restoration: Numerical Methods and Extensions, Proceedings International Conference on Image Processing, ICIP-97, October 26-29, 1997, Santa Barbara, California, Vol. III, 384-387.

[13] M.G. Crandall and T.M. Liggett, Generation of Semigroups of Nonlinear Transformations on General Banach Spaces, Amer. J. Math., 93 (1971), 265-298.

[14] F. Dibos and G. Koepfler, A method to minimize total variation, preprint.

[15] L.C. Evans and R.F. Gariepy, "Measure Theory and Fine Properties of Functions," Studies in Advanced Math., CRC Press, 1992.

[16] P.L. Lions, S. Osher and L. Rudin, Denoising and Deblurring using Constrained Nonlinear Partial Differential Equations, Tech. Repport, Cognitech Inc., Santa Monica, CA, 1992, submitted to SINUM. 
[17] L. Rudin and S. Osher, Total Variation based Image Restoration with Free Local Constraints, Proc. of the IEEE ICIP-94, vol. 1, Austin, TX, 1994, 31-35.

[18] L. Rudin, S. Osher and E. Fatemi, Nonlinear Total Variation based Noise Removal Algorithms, Physica D., 60 (1992), 259-268.

[19] G. Sapiro and V. Caselles, Histogram Modification via Differential Equations, Journal of Differential Equations, 135 (1997), 238-268.

[20] G. Sapiro and A. Tannenbaum, On Affine Plane Curve Evolution, Journal of Functional Analysis, 119:1 (1994), 79-120.

[21] D. Strong and T. Chan, Exact Solutions to Total Variation Regularization Problems, CAM Report 96-41, UCLA, October 1996.

[22] C.R. Vogel and M.E. Oman, Iterative Methods for Total Variation Denoising, SIAM J. Sci. Computing, to appear.

[23] C.R. Vogel and M.E. Oman, Fast total variation based image reconstruction, Proceedings of the 1995 ASME Design Engineering Conferences, Vol. 3 (1995), 1009-1015.

[24] W.P. Ziemer, "Weakly Differentiable Functions," GTM 120, Springer Verlag, 1989. 


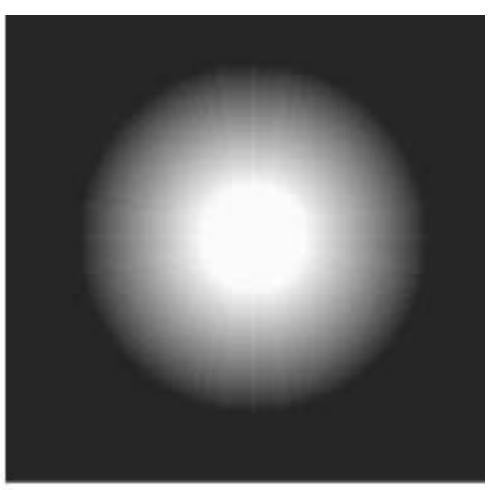

Figure 1.1.

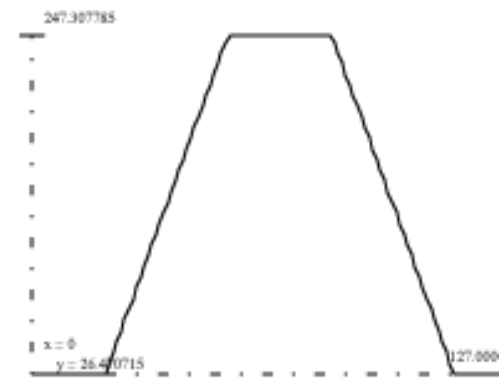

Figure 1.3.

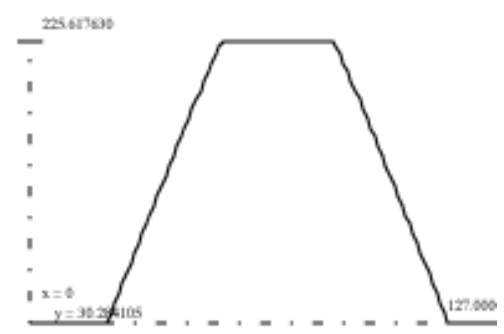

Figure 1.5.

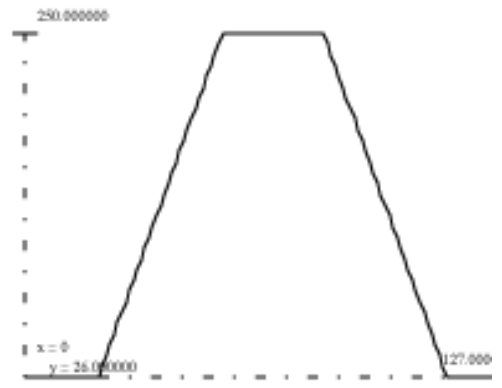

Figure 1.2.

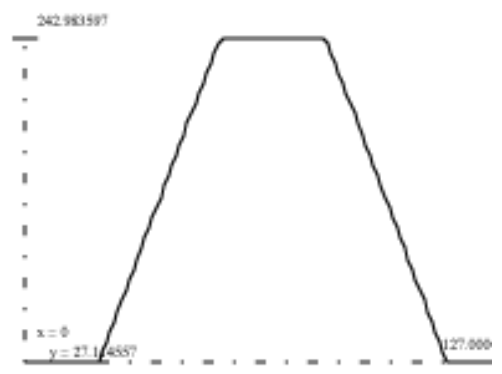

Figure 1.4.

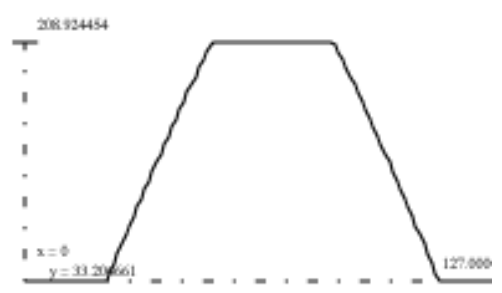

Figure 1.6.

Figure 1. Fig. 1.1: original image. Fig. 1.2: its corresponding sectional view at $x_{1}=64, x_{2} \in[0,127]$. Fig. 1.3 to 1.6 : same sectional view of the solution after 100, 200, 600 and 1000 time steps, respectively. 


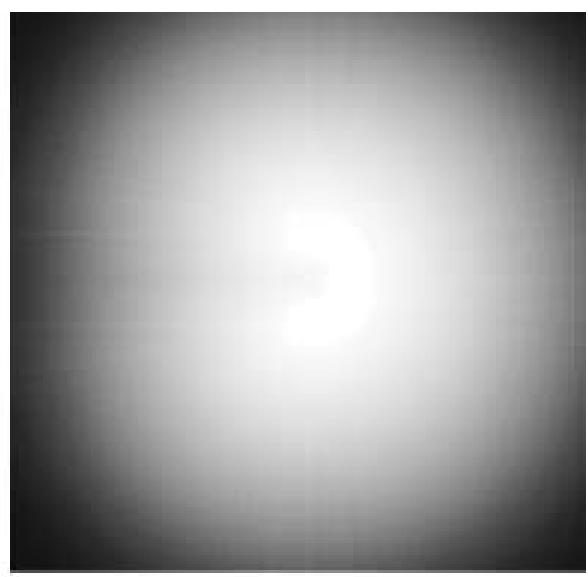

Figure 2.1.

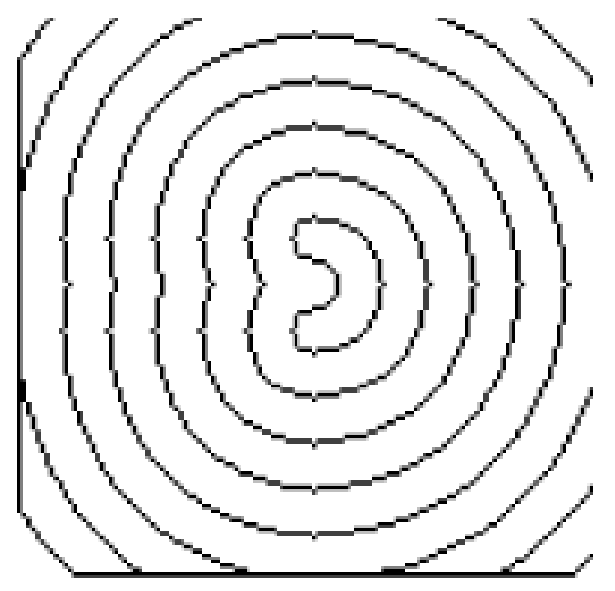

Figure 2.3.

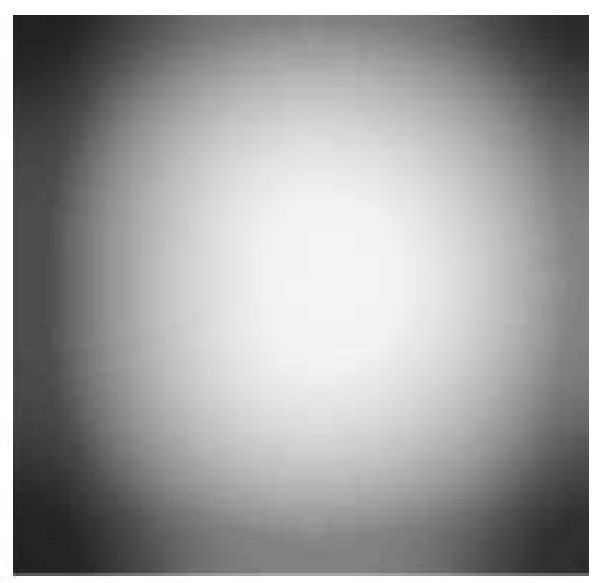

Figure 2.2.

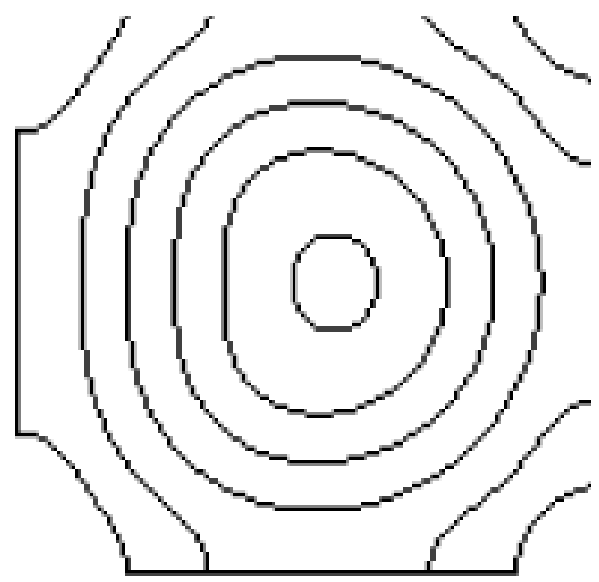

Figure 2.4. 


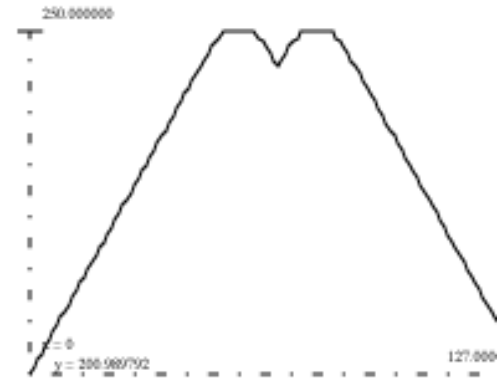

Figure 2.5.

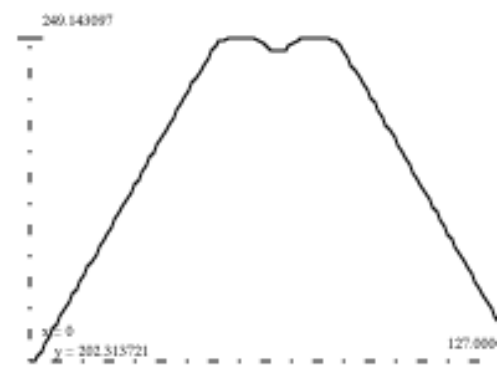

Figure 2.7.

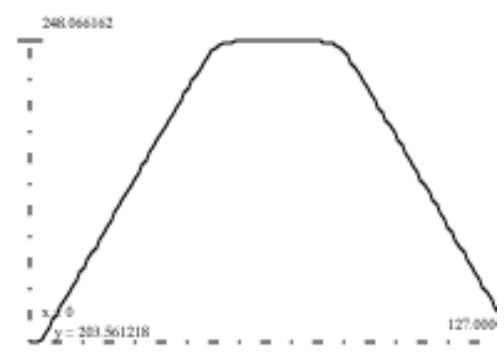

Figure 2.9.

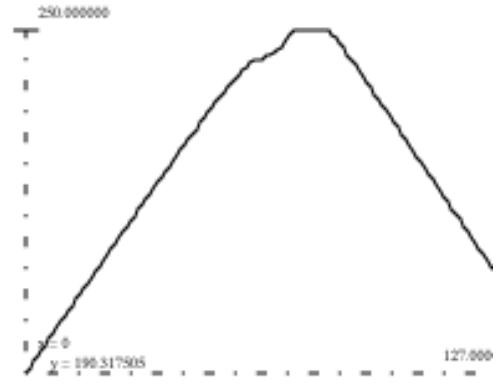

Figure 2.6.

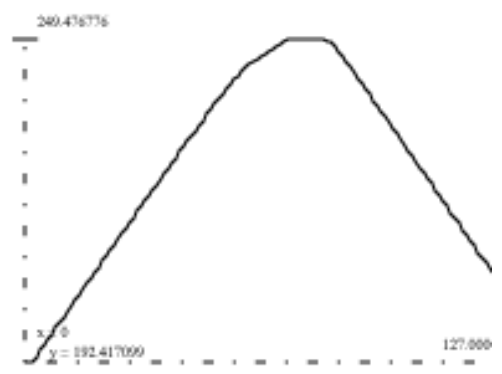

Figure 2.8.

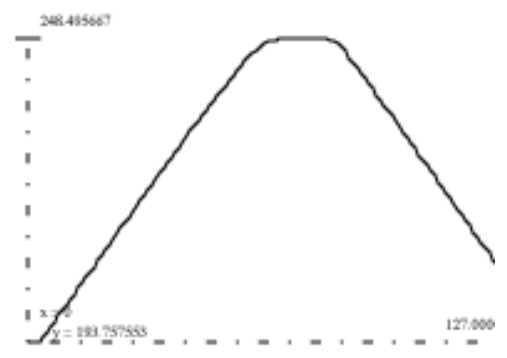

Figure 2.10.

Figure 2. Fig. 2.1: original image. Fig. 2.2: solution after 100 time steps. Fig. 2.3 and 2.4: level lines of images in Fig. 2.1 and 2.2, respectively, taken at multiples of 10 . Fig. 2.5 and 2.6: sectional views of the original image corresponding to $x_{1}=64, x_{2} \in[0,127]$ (left) and to $x_{2}=64$, $x_{1} \in[0,127]$ (right). Fig. 2.7 to 2.10: same sectional views of the solution after 10 and 25 time steps, respectively. 


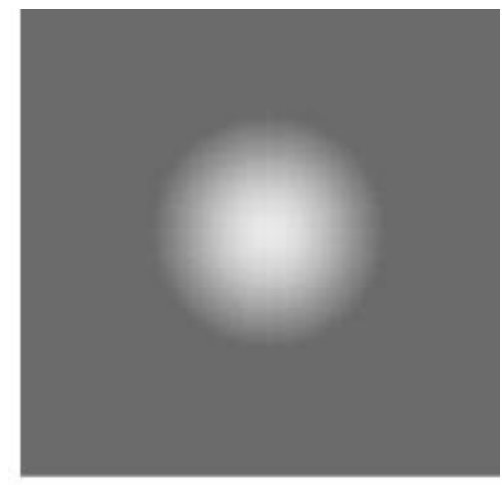

Figure 3.1.

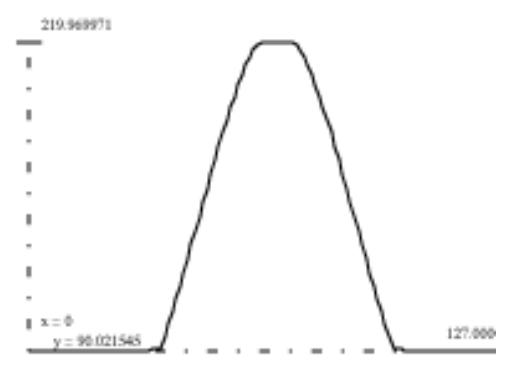

Figure 3.3.

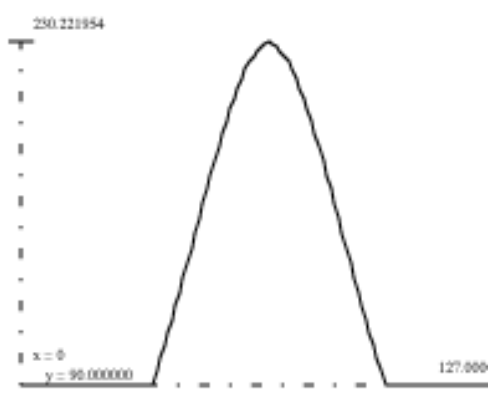

Figure 3.2.

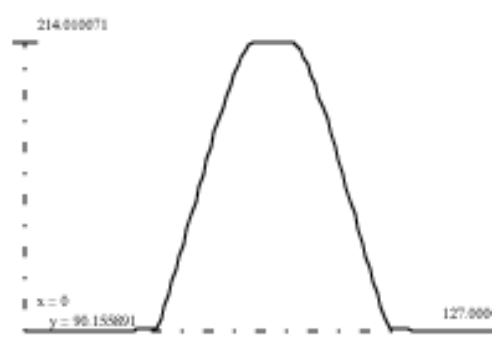

Figure 3.4.

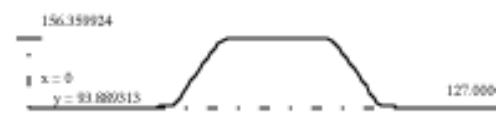

Figure 3.5.

Figure 3. Fig. 3.1: original image. Fig. 3.2: its corresponding sectional view at $x_{1}=64, x_{2} \in[0,127]$. Fig. 3.3 to 3.5 : same sectional view of the solution after 50, 100 and 1000 time steps, respectively. 


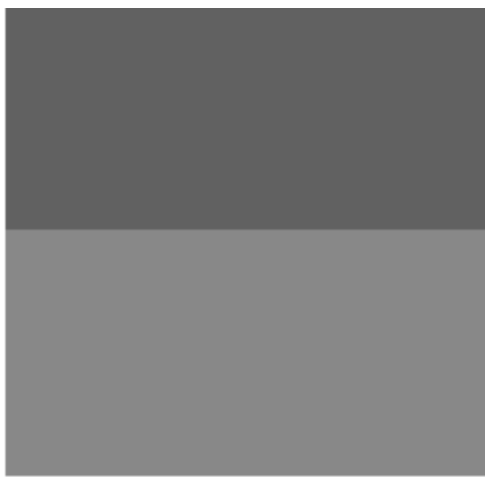

Figure 4.1.

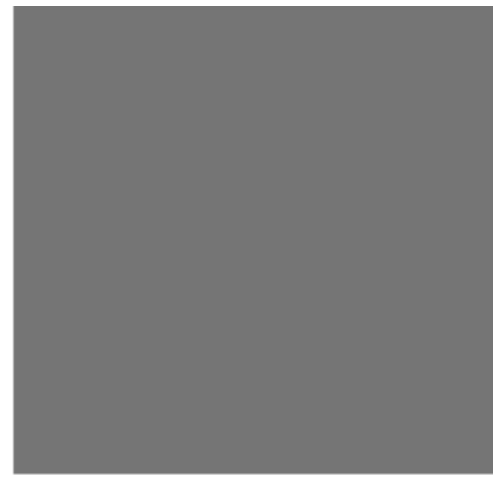

Figure 4.2.

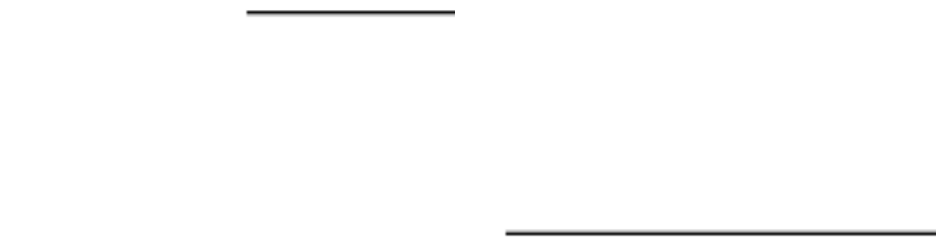

Figure 4.3.

Figure 4.4.

Figure 4. Fig. 4.1: original image. Fig. 4.2 solution after 3000 time steps. Fig. 4.3 and 4.4: sectional view of Fig. 4.1 and 4.2 corresponding to $x_{1}=20, x_{2} \in[0,39]$. 